\title{
Micromachined Arrayed Capacitive Ultrasonic Sensor/Transmitter with Parylene Diaphragms
}

\author{
Seiji Aoyagi \\ Kansai University \\ Japan
}

\section{Introduction}

For the external environment recognition of a robotic field, an ultrasonic sensor has advantages in cost performance compared with other sensors such as vision devices. In particular, in the spaces where vision devices cannot be used (e.g., in the dark, smoky situation such as in the disaster site), ultrasonic sensors are effective. For the purpose of using ultrasonic devices in microrobot applications (Aoyagi, 1996), and/or for the purpose of imitating the dexterous sensing functions of animals such as bats and dolphins (Mitsuhashi, 1997; Aoyagi, 2001), it is necessary to miniaturize the current ultrasonic sensors/transmitters (Haga et al., 2003).

The effectiveness of miniaturization is discussed herein from the viewpoint of directivity. Let us assume a piston-type ultrasonic device, the radius of which is $R$. The angle $\theta_{1 / 2}$ at which the sound pressure level becomes half of the maximal level achieved on the centerline of the piston $(\theta=0)$ is expressed as follows (Mitsuida, 1987):

$$
\theta_{1 / 2}=\sin ^{-1}(0.353 \lambda / R)
$$

where $\lambda$ is the wavelength. The schematic explanation of this angle is shown in Fig. 1. This equation indicates that directivity becomes wider as the radius becomes smaller. Using many miniaturized transmitters/sensors in an array, the electrical scanning of directivity based on the delay-and-summation principle (Fig. 2) (Ono et al., 2005; Yamashita et al., 2002a; Yamashita et al., 2002b) and acoustic imaging based on the synthesis aperture principle (Guldiken \& Degertekin, 2005) are possible, which could be effectively used for robotic and medical applications. Miniaturizing one sensing/transmitting element is useful both for realizing an arrayed device in a limited space and for realizing a device with omnidirectional characteristics, since the directivity of each element becomes wider as its diaphragm area becomes smaller based on equation (1).

There are two types of available ultrasonic sensor, one is piezoelectric, and another is capacitive. The working principle and the typical received waveform of piezoelectric type are schematically shown in Fig. 3. This type is further classified to thin film type and bimorph type. The former uses a micromachined thin film as a diaphragm, on which piezoelectric material such as lead zirconate titanate (PZT) is deposited using sol-gel method or sputtering. The latter uses a rather thick bulk plate as an elastic body of receiving and/or transmitting ultrasound. In case of the thin film type, piezoelectric constant $d_{31}$ is rather 
small, so it can act only as a receiver and cannot transmit ultrasound. Although the bimorph type can transmit ultrasound, its size is comparatively large.

The merit of these piezoelectric types is that they do not require bias voltage for their operation. The drawback of piezoelectric types is that the received waveform is burst one, i.e., the waveform continues during several tens cycles, since they are usually operated at their resonant frequencies with small damping. In the ranging system for airborne use (see Section 4.5), the precise arrival time of the ultrasound is difficult to detect for the burst waveform with dull rising, since the first peak is difficult to detect by setting a threshold level.

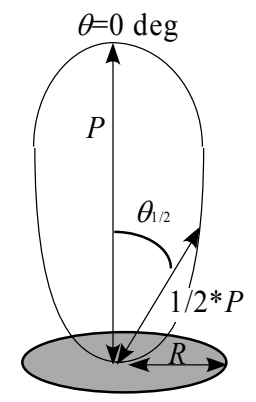

Fig. 1. Definition of $\theta_{1 / 2}$.

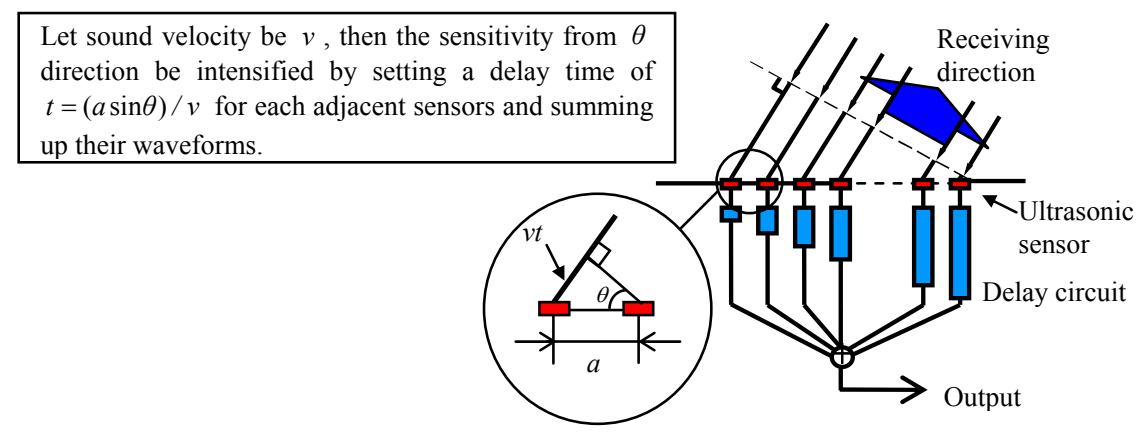

Fig. 2. Electrical scanning of directivity.

By contrast, although the capacitive type needs bias voltage for its operation, it can detect the arrival time of ultrasound accurately by setting an appropriate threshold level, since the received waveform is impulsive and well-damped, as schematically shown in Fig.4. A capacitive sensor can also act as a transmitter by applying an impulsive high voltage between two electrodes (Sasaki \& Takano, 1988; Diamond et al., 2002), i.e., a diaphragm and a backing plate, both of which are conductive or coated by thin metal films.

As an example of conventional commercially available capacitive microphones, B\&K-type 4138 (Brüel \& Kjær, 1982) can receive sound pressure in the ultrasonic frequency range, and can be approximated to be nondirectional by virtue of the small area of its diaphragm. The structure of this microphone is shown in Fig. 5. The diameter, sensitivity, and frequency bandwidth of this microphone are $1 / 8$ in. (3.175 mm), $0.9 \mathrm{mV} / \mathrm{Pa}$, and $100 \mathrm{kHz}$, respectively. However, this microphone has the drawback of being expensive due to its 
complicated and precise structure, i.e., it is composed of a thin nickel diaphragm of $1.6 \mu \mathrm{m}$ thickness, a support rim, and a nickel backing plate facing the diaphragm surface with a small gap of $20 \mu \mathrm{m}$.

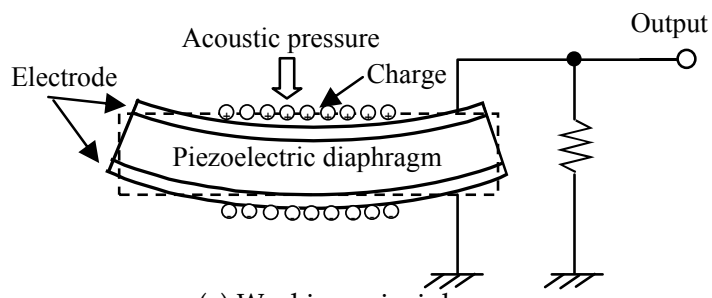

(a) Working principle

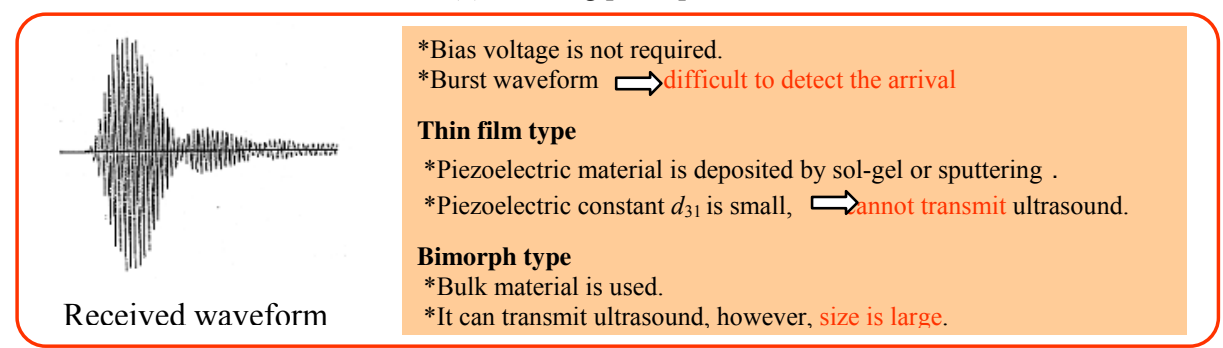

(b) Typical received waveform

Fig. 3. Piezoelectric type ultrasonic sensor.

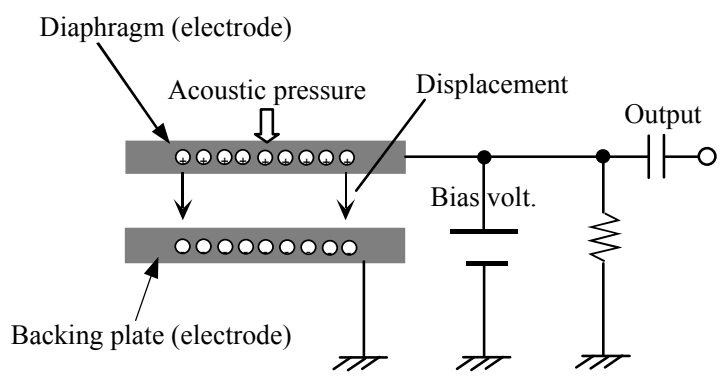

(a) Working principle

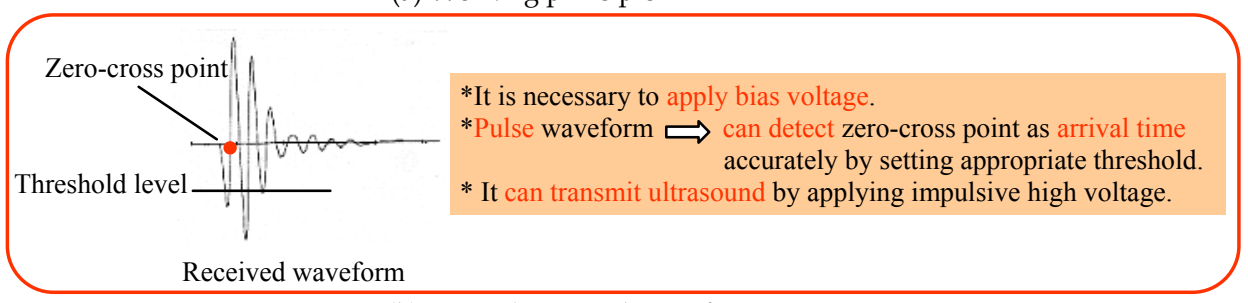

(b) Typical received waveform

Fig. 4. Capacitive type ultrasonic sensor. 
A capacitive sensor can also transmit ultrasound by applying impulsive high voltage as mentioned above: however, this B\&K microphone is not applicable for the use of a transmitter because of the possibility of diaphragm fracture, taking into account its high cost.

In contrast, several studies on a capacitive microphone with a silicon diaphragm (Scheeper et al., 1992; Bergqvist \& Gobet, 1994; Ikeda et al., 1999; Chen et al., 2002; Martin et al., 2005; Khuri-Yakub et al., 2000; Zhuang et al., 2000) have been conducted using micromachining technology (Kovacs, 1998), and some of them have been commercialized (Knowles Acoustics, 2002). Using this technology, numerous arrayed miniaturized ultrasonic sensors with uniform performance can be fabricated on a silicon wafer with a fine resolution of several microns and a comparatively low cost, which may make it possible to fabricate an arrayed-type sensor (Yamashita et al., 2002a; Yamashita et al., 2002b; Guldiken \& Degertekin, 2005; Khuri-Yakub et al., 2000; Zhuang et al., 2006) and to activate it as a transmitter or speaker (Diamond et al., 2002; Khuri-Yakub et al., 2000).

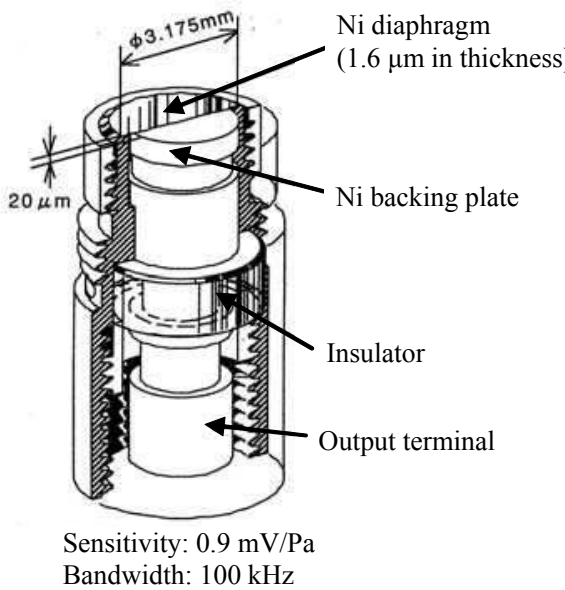

B\&K type 4138 is one of few commercial condenser type microphones, which can receive sound pressure of ultrasonic frequency range and can achieve comparatively wide directivity based on its small size.

Drawbacks

expensive caused by its complicated and precise structure

not used as transmitter, restricted from fracture possibility of the diaphragm, taking account of its expensive cost

Fig. 5. Stracture of Brüel \& Kjær 4138 microphone.

In micromachined capacitive microphones, the diaphragms are generally made of a siliconbased material, such as polysilicon and silicon nitride. In a few studies a polymer material was used for the diaphragms, such as polyimide (Pederson et al., 1998; Schindel et al., 1995), poly(tetrafluoroethylene) (trade name: Teflon) (Hsieh et al., 1999), and poly(ethylene terephthalate) (PET; trade name: Mylar) (Schindel et al., 1995). Since polymer materials have high durability due to their flexibility and nonbrittleness compared with silicon-based materials, their use in transmitters or speakers is thought to be possible. That is, the possibility of survival of a polymer diaphragm would be higher compared with that of a silicon diaphragm even when the applied high impulsive voltage for transmission passes instantaneously over the collapse voltage (Yaralioglu et al., 2005), at which the diaphragm is strongly pulled by an electrostatic attractive force to adhere to the substrate, causing the collapse of the device structure. Since a large displacement of the diaphragm per sound pressure is obtained due to the flexibility of the polymer diaphragm, the high sensitivity of the microphone can be realized. This is because the mechanical impedance of the diaphragm theoretically becomes low as the Young's modulus of the diaphragm's material decreases, 
provided that the radius, thickness, and input frequency are constant (Khuri-Yakub et al., 2000).

An ultrasonic transducer with a Mylar diaphragm has been commercialized (MicroAcoustic Instruments, trade name: BAT), and is often used in the ultrasonic research field (Hayashi et al., 2001); however, although the pits on the backing plate of this transducer are fabricated by micromachining technology, the polymer diaphragm film is assembled by pressing it to the backing plate with adequate pre-tension using a holder, the assembly of which appears as complicated as that of the above-mentioned B\&K-type 4138 microphone.

Polyparaxylene (trade name: Parylene) is one of the polymer materials expected to be applied in the polymer micro-electro-mechanical-systems (MEMS) field (Tai, 2003). The deposition of Parylene is based on chemical vapor deposition (CVD), which is suitable for MEMS diaphragm fabrication. The mechanical properties of silicon, silicon nitride, Parylene, and Mylar are compared, as shown in Table 1. In addition to its flexible and nonbrittle characteristics compared with common polymer materials, Parylene has several excellent characteristics as follows. 1) It is a biocompatible material, which allows medical applications of the device. 2) It is chemically stable, i.e., it has high resistivity to acid, base, and organic solvents, which protects the device from external chemical environments. 3) It has high complementary metal oxide semiconductor (CMOS) compatibility compared with other polymer materials, since it can be deposited at room temperature. This characteristic makes the integration of a device with electrical circuits possible; such a device is called a smart device. 4) Its CVD deposition is conformal, thus the deposition of a domeshaped diaphragm is possible, which is effective for realizing a real spherical sound source/receiver. Due to these characteristics, an ultrasonic device utilizing a Parylene diaphragm has great potential in future applications. The principal aim of this study is to develop a capacitive microphone with a Parylene diaphragm (Aoyagi et al., 2007a).

\begin{tabular}{ccccc}
\hline & $\begin{array}{c}\text { Young's modulus } \\
(\mathrm{GPa})\end{array}$ & $\begin{array}{c}\text { Shear modulus } \\
(\mathrm{GPa})\end{array}$ & $\begin{array}{c}\text { Density } \\
\left(\mathrm{kg} / \mathrm{m}^{3}\right)\end{array}$ & Poisson ratio \\
\hline Silicon $^{* 1}$ & 131 & 80 & 2,330 & 0.27 \\
Silicon nitride $^{* 2}$ & 290 & - & 3,290 & 0.27 \\
Parylene & 3.2 & - & 1,287 & 0.4 \\
PET (Mylar) & 2.8 & - & 1,370 & 0.4 \\
\hline
\end{tabular}

*1 Crystal silicon in (100) plane.

*2 LP CVD $\mathrm{Si}_{3} \mathrm{~N}_{4}$ (Tabata et al., 1989).

- Not cleared.

Table 1. Comparison of mechanical properties of silicon and polymer materials.

The reported capacitive microphones focus on audio applications, in which bandwidth is below $15-20 \mathrm{kHz}$, where the important issues include sensitivity, linearity, and noise floor. In contrast, the present Parylene transducer focuses on ultrasonic applications in air, in which bandwidth is as high as $100 \mathrm{kHz}$, where the important issue is the accuracy of the distance measurement between the transmitter and the receiver. The directivity of the sensor is also the important issue in these applications. The second aim of this research is to characterize the fabricated Parylene ultrasonic receiver from the viewpoints of the accuracy of distance measurement and the directivity (Aoyagi et al., 2007a). 
As the third aim of this research, an arrayed sensor device comprising $5 \times 5$ developed sensors is fabricated, and its receiving performance is characterized to prove the possibility of the electrical scanning of directivity based on delay-and-summation principle (Aoyagi et al., 2008a). As the fourth aim of this research, we confirm that each developed sensor can act as a transmitter by applying a high impulsive voltage, which means that the scanning of transmitting directivity is also possible. In this research, the scanning performance as the arrayed transmitter is also characterized (Aoyagi et al., 2008b).

\section{Structure design of a sensor with Parylene diaphragm}

\subsection{Resonant frequency considering intrinsic stress}

The resonant frequency of a Parylene diaphragm is investigated to define the size of the sensor and the bandwidth herein. The shape of the diaphragm is assumed to be a circle. Since Parylene has intrinsic tensile stress influenced by the temperature history of the fabrication (Harder et al., 2002), the relationship between the tensile stress and the resonant frequency is investigated herein.

Assume that the diaphragm has membrane characteristics, in which internal tensile stress plays an important role. Then, the following theoretical expression exists according to the theory of elastic vibration (Sato et al., 1993):

$$
\omega_{n}=\lambda_{n s} \frac{1}{R} \sqrt{\frac{\sigma}{\rho}},
$$

where $\omega_{n}$ is the resonant frequency $(\mathrm{rad} / \mathrm{s}), \lambda_{n s}$ is the eigenvalue (2.405), $\sigma$ is the intrinsic tensile stress in the diaphragm $\left(\mathrm{N} / \mathrm{m}^{2}\right), \rho$ is the density of the diaphragm material $\left(\mathrm{kg} / \mathrm{m}^{3}\right)$, and $R$ is the radius of the diaphragm $(\mathrm{m})$.

In FEM (Finite Element Method) simulation, $\sigma$ is applied in the cross section area of the boundary, i.e., the rim, which stretches the diaphragm. The modal FEM simulation is carried out for this stretched diaphragm. ANSYS is employed as the FEM software. In case the diaphragm radius $R$ is $500 \mu \mathrm{m}$, theoretical and FEM simulated values of resonant frequency are obtained by changing the value of tensile stress in the range of 0-30 MPa. The result is shown in Fig. 6 . This result shows that the influence of tensile stress on the resonant frequency is large. In the following part of this paper, it is assumed that the tensile stress $\sigma$ is $25 \mathrm{MPa}$, based on the experimental data using rotation tip measurement (see Section 3.2). Under this condition, the relationship between the radius and the resonant frequency is shown in Fig. 7. Considering that the aimed bandwidth is in the ultrasonic range of 40-100 $\mathrm{kHz}$, a radius $R$ in the range of $500-1,200 \mu \mathrm{m}$ is employed in this research according to this figure.

\subsection{Influence of acoustic holes on damping ratio}

In microphones, acoustic holes are generally set in the backing plate to control air damping. In the case of a simple square diaphragm, the viscous damping coefficient is calculated analytically (Scheeper et al., 1992; Bergqvist \& Gobet, 1994; Škvor, 1967) in relation to the number of acoustic holes and to the surface fraction occupied by the acoustic holes. However, there has been no research on air damping for an arbitrary diaphragm shape. Thus, the damping ratio of a circular diaphragm is simulated using the FEM software. 


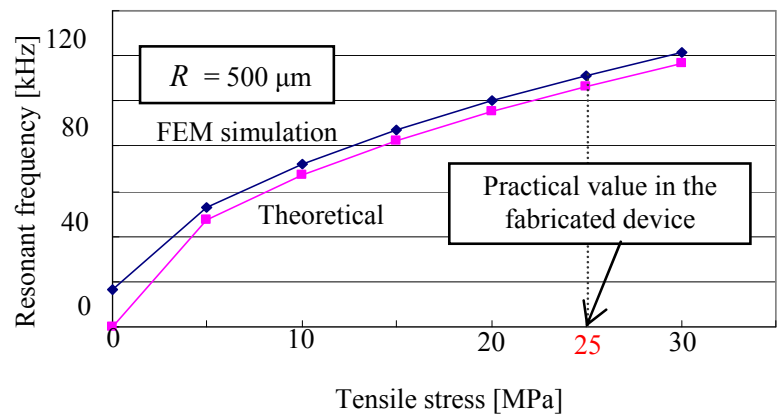

Fig. 6. Relationship between tensile stress and resonant frequency.

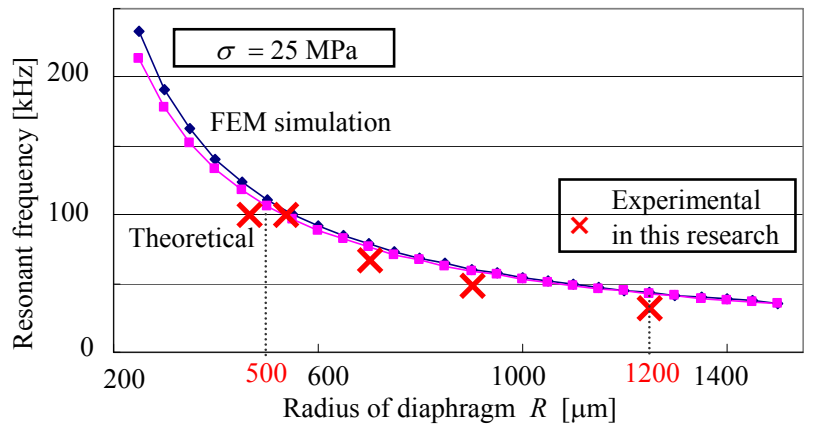

Fig. 7. Relationship between diaphragm radius and resonant frequency.

The flow distribution inside the air gap between the diaphragm and the backing plate, and the flow distribution inside the acoustic holes are simulated by FEM. Taking symmetry into account, a quarter model is employed. An example of the simulation model and its result are shown in Fig. 8. The transition of the displacement distribution, which is based on the first-order resonant vibration mode of a circular diaphragm, was given to the diaphragm. Then, the distribution of vertical flow velocity under the diaphragm was simulated. Total force $F$ was obtained by summing up the pressures of all the elements just below the diaphragm. Flow velocity $u^{*}$ was obtained by averaging the velocities of all the elements inside the air gap. Then, the damping ratio $\zeta$ was obtained as follows:

$$
\zeta=\frac{\lambda}{2 m \omega_{n}}=\frac{F / u^{*}}{2 m \omega_{n}}
$$

where $m$ is the mass of the diaphragm, $\omega_{n}$ is the resonant frequency of the diaphragm, $\lambda$ is the viscous damping coefficient.

The effects of the radius of the acoustic hole $r$ and the number of holes $n$ on the damping ratio $\zeta$ were investigated. The simulation result is shown in Fig. 9. Three cases in which the 
radii of the diaphragm $(R)$ were 500,700 , or 1,200 $\mu \mathrm{m}$ are focused on. Considering the practical fabrication condition, the air gap and thickness of the backing plate are assumed to be 1.5 and $150 \mu \mathrm{m}$, respectively.

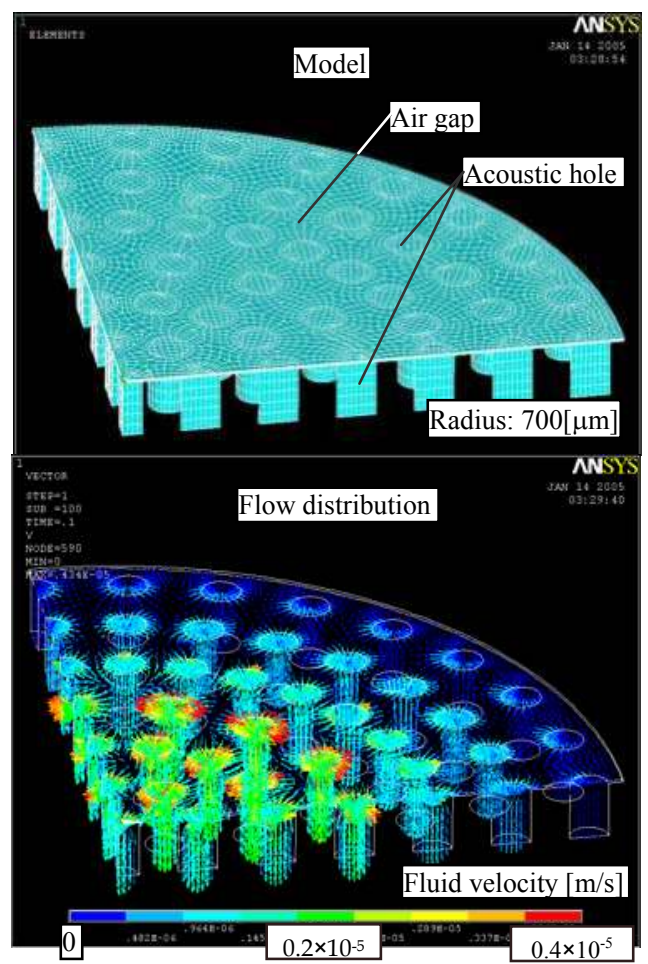

Fig. 8. FEM simulation for influence of acoustic holes on damping ratio.

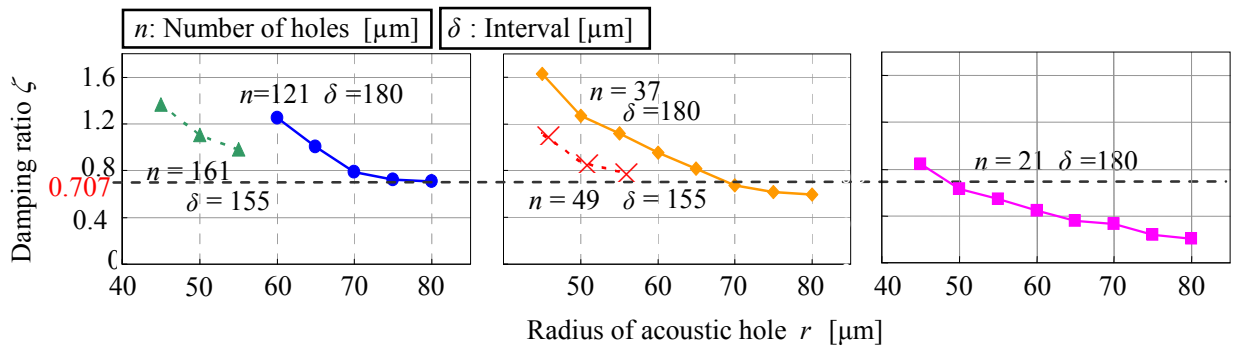
(a) $R=1200 \mu \mathrm{m}$
(b) $R=700 \mu \mathrm{m}$
(c) $R=500 \mu \mathrm{m}$

Fig. 9. Damping ratio by FEM simulation.

Also, considering the practical fabrication condition, several combinations of $r$ and $\delta$ (the interval of adjacent acoustic holes) are tested to realize the optimal damping ratio of $\zeta=1 / \sqrt{2}=0.707$ through trial and error. 
In this figure, the damping ratio $\zeta$ is inversely proportional to $r$ and $n$. Also, $\zeta$ decreases as $R$ decreases, indicating that air damping is less effective for smaller diaphragms. For example, in the case of $R=1,200 \mu \mathrm{m}$, the condition in which $n=121$ and $r=80 \mu \mathrm{m}$ with $\delta=$ $180 \mu \mathrm{m}$ is suitable for realizing the optimal damping ratio. Photomasks for a micromachining fabrication of the sensor structure including acoustic holes are designed on the basis of the simulation results explained herein.

\section{Fabrication process of a sensor}

\subsection{Fabrication process}

The ultrasonic sensor was fabricated by depositing Parylene ( $2 \mu \mathrm{m}$ in thickness) on a Si wafer $(150 \mu \mathrm{m}$ in thickness) with a thermally grown oxide $(1 \mu \mathrm{m}$ in thickness). Parylene deposition was based on chemical vapor deposition (CVD), and a coating apparatus (PDS2010, Specialty Coating Systems) was used. The schematic overview of the developed sensor is shown in Fig. 10. The process flow is shown in Fig. 11 and proceeded as follows:

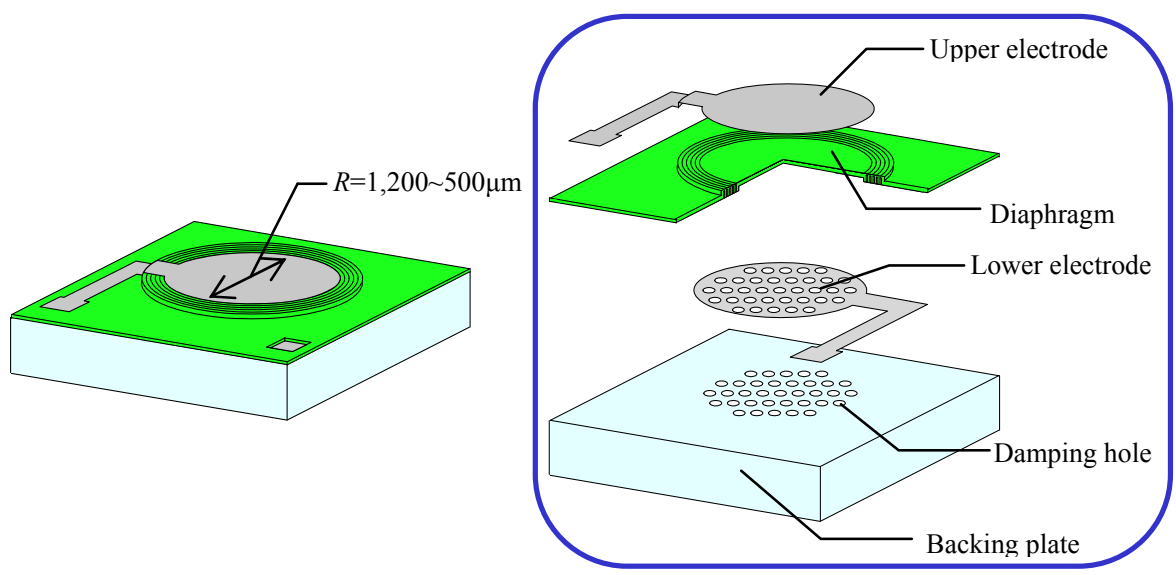

Fig. 10. Schematic overview of parylene ultrasonic sensor.

Aluminum ( $0.2 \mu \mathrm{m}$ in thickness) was sputtered onto the oxidized silicon wafer, and patterned for the lower electrode and the bonding pad (see Fig. 11(1)).

As a sacrificial layer, amorphous silicon (1.5 $\mu \mathrm{m}$ in thickness) was deposited by plasmaenhanced CVD, followed by etching using $\mathrm{SF}_{6}$ plasma to make slots, the function of which is explained later (see Fig. 11(2)).

The Parylene ( $2 \mu \mathrm{m}$ in thickness) layer was deposited and patterned using $\mathrm{O}_{2}$ plasma to reveal a bonding pad area (see Fig. 11(3)). In this patterning, a photoresist of $5 \mu \mathrm{m}$ (AZP4903) was used as the etching mask. Since the etching ratios of Parylene and the photoresist are almost the same, the mask made of the photoresist is gradually consumed during $\mathrm{O}_{2}$ plasma etching. Therefore, a rather thick photoresist was employed.

The slots on the amorphous Si layer were filled with Parylene, providing anchor contact between Parylene and the substrate. Considering the mechanical strength at the edge of the diaphragm, it is desirable that the height of Parylene is the same at the anchor and the diaphragm. If the anchor contact area is large, the height of Parylene at the anchor will be smaller than that at the diaphragm by the thickness of the sacrificial layer, as schematically 
shown in Fig. 12(a). To cope with this problem, slots were created and the anchor contact area was minimized. The height of the anchor was maintained at the same level as that of the diaphragm, since Parylene deposition is so conformal as to fill up these slots, as schematically shown in Fig. 12(b). The shapes and sizes of the slots for the anchor are shown in Fig. 12(c).

Aluminum (0.5 $\mu \mathrm{m}$ in thickness) was sputtered and patterned for the upper electrode using the liftoff process. This electrode must surpass the step height of Parylene and amorphous silicon layer (totally $3.5 \mu \mathrm{m}$ in thickness) to reach the bonding pad, so a comparatively thick aluminum layer is necessary (see Fig. 11(4)).

The backside of the silicon wafer was dry etched by Inductively-Coupled Plasma Deep Reactive Ion Etching (ICP-DRIE) to produce acoustic holes (see Fig. 11(5)). These holes also play a role as the etching holes for the sacrificial amorphous silicon layer, inside which $\mathrm{XeF}_{2}$ etching gas was later introduced.

The oxide layer at the bottom of the acoustic holes was etched using $\mathrm{CHF}_{3}$ plasma (see Fig. 11(6)). The sidewalls of the acoustic holes were covered by Parylene ( $1 \mu \mathrm{m}$ in thickness) to protect them from the $\mathrm{XeF}_{2}$ etching gas used later. The conformal deposition of Parylene assists this process (see Fig. 11(7)). The Parylene at the bottom of the holes was etched using $\mathrm{O}_{2}$ plasma. The vertical etching characteristic of the reactive ion etching (RIE) assists the selective etching of the bottom area.

$-\mathrm{SiO}_{2} \bigotimes \mathrm{Al} \bigcirc \underset{\text { (amorphous silicon) }}{\mathrm{a}-\mathrm{Si}}$ Parylene

(1)

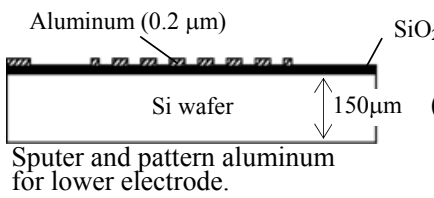

Anchor contact area

(2)
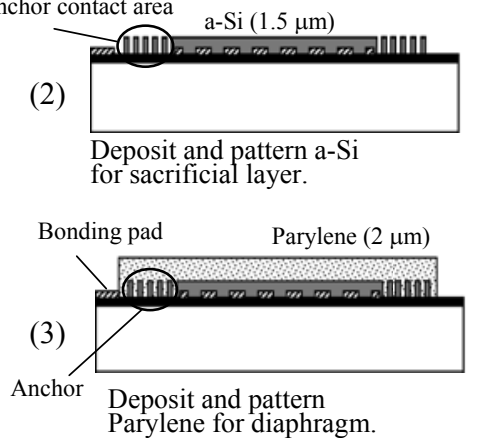

(5)

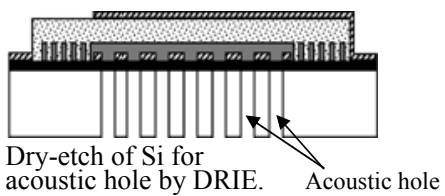

(6)

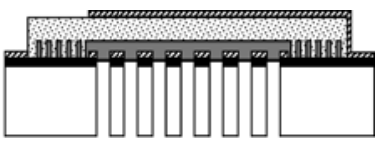

Dry-etch of oxide by RIE.

(7)

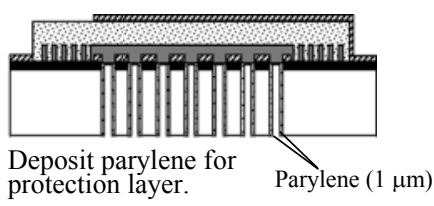

(4)

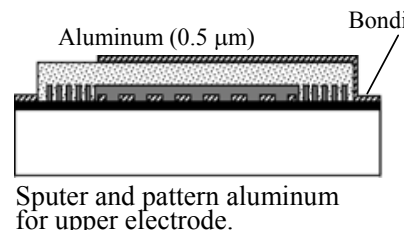

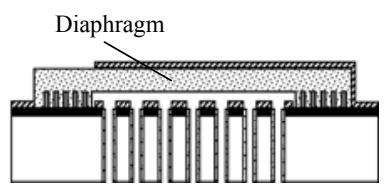

Dry-etch of a-Si by $\mathrm{XeF}_{2}$ for releasing diaphragm.

Fig. 11. Process flow of ultrasonic sensor. 
Finally, the sacrificial amorphous silicon layer was dry etched away using $\mathrm{XeF}_{2}$ gas in order to release the diaphragm (see Fig. 11(8)). This dry etching process is effective for preventing stiction (Yao et al., 2001).

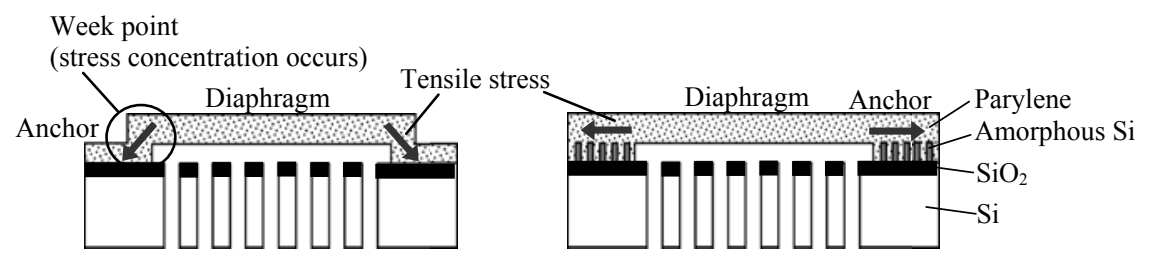

(a) without slots

(b) with slots

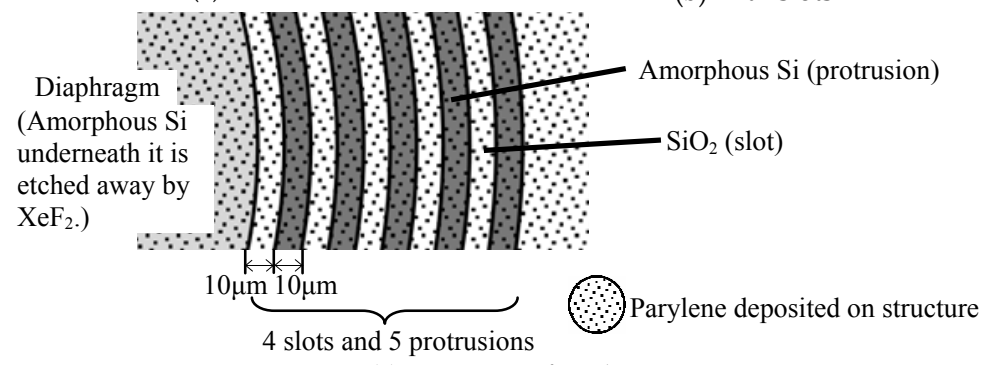

(c) top wiew of anchor

Fig. 12. Reducton of stress concentration using slots.

\subsection{Fabrication results and intrinsic stress}

An overview and schematic cross section of the fabricated sensor are shown in Fig. 13. Scanning Electron Microscope (SEM) images of fabricated sensors are shown in this figure. In this example, the radius of the diaphragm is $1,200 \mu \mathrm{m}$, and that of the acoustic hole is $50 \mu \mathrm{m}$.

Looking at the back-side and cross section views of SEM images, it is proven that the acoustic holes were successfully fabricated. In the front-side view of SEM image, the Parylene circular diaphragm over the acoustic holes is seen. The aluminum upper electrode crossing the anchor is seen.

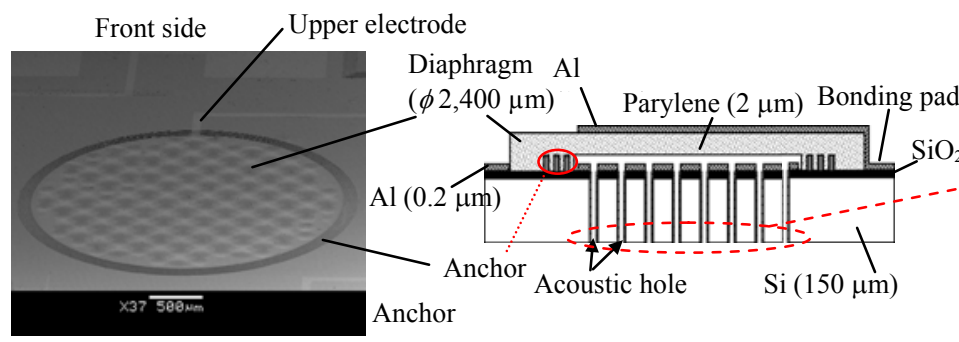

Amorphous silicon is used as sacrificial layer, which is dryetched away by $\mathrm{XeF}_{2}$.

The radius and number of acoustic holes are determined by FEM in order to achieve adequate damping.

Fig. 13. Overview and schematic cross section of fabricated sensor. 
A rotation tip was fabricated in the same substrate in order to estimate the actual tensile stress of Parylene, as shown in Fig. 14. The shrinkage of the beams supporting the tip is $H \cdot \tan \alpha$, and the strain in the film is calculated as $H \cdot \tan \alpha /\left(L_{A}+W+L_{B}\right)$, using symbols in Fig. 14. Multiplying the strain by Young's modulus of Parylene (3.2 GPa), the stress is obtained, which is proven to be approximately $25 \mathrm{MPa}$.

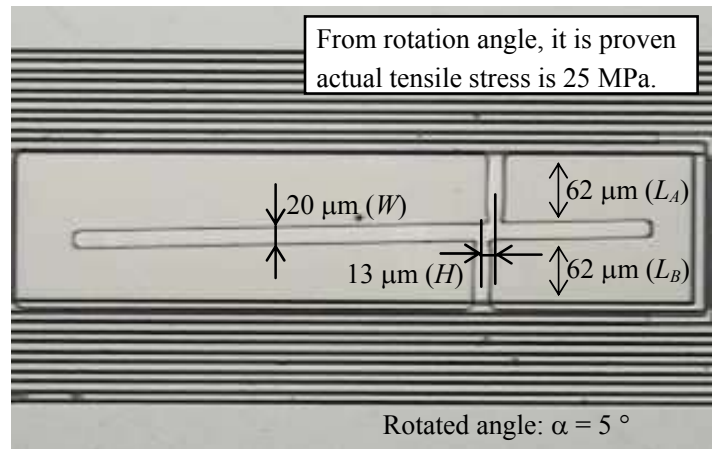

Fig. 14. Optical image of rotation tip.

\section{Receiving performance of a sensor}

\subsection{Detecting circuitry for capacitance change}

The circuitry used to detect the capacitance change due to the diaphragm displacement caused by ultrasonic sound pressure is documented herein. A bias voltage of $100 \mathrm{~V}$ was applied to the fabricated Parylene capacitive sensor. This value has an effect on the sensitivity, resonant frequency, and bandwidth (Schindel et al., 1995; Yaralioglu et al., 2005). In this study, this value is defined on the basis of values in references, in which $150 \mathrm{~V}$ (Sasaki et al., 1988), $100 \mathrm{~V}$ (Khuri-Yakub et al., 2000), 100-400 V (Schindel et al., 1995), and 50-135 V (Yaralioglu et al., 2005) were employed. In this study, the values of 150 and $200 \mathrm{~V}$ were experimentally tested; however, it was observed that the diaphragm was broken when a high impulsive voltage of $700 \mathrm{Vpp}$ was applied during the transmitter use (the detail of which is explained in Section 6), although this failure rate is small. Thus, considering the safety factor, the value of $100 \mathrm{~V}$ was employed, under which condition neither diaphragm failure nor the disconnection of wiring was encountered.

Upon being supplied with a constant electrical charge due to the bias voltage, the diaphragm displacement was transformed to the voltage change at the sensor's electrode, and it was amplified by a factor of $30(29.5 \mathrm{~dB})$. The circuitry used for capacitance-to-voltage (CV) transformation and amplification is shown in Fig. 15, in which the high-frequency component of the voltage change is extracted by a bias-cut condenser, and it is input to an operational amplifier by a shunt resistor. Only the range within $\pm 0.7 \mathrm{~V}$ is dealt with for amplification by virtue of a voltage limiter using two diodes, considering noise reduction.

\subsection{Experimental setup for characterizing receiving performance}

The experimental setup for characterizing the receiving performance of the developed sensor is schematically shown in Fig. 16. An electric spark discharge was used as an ultrasonic transmitter. 


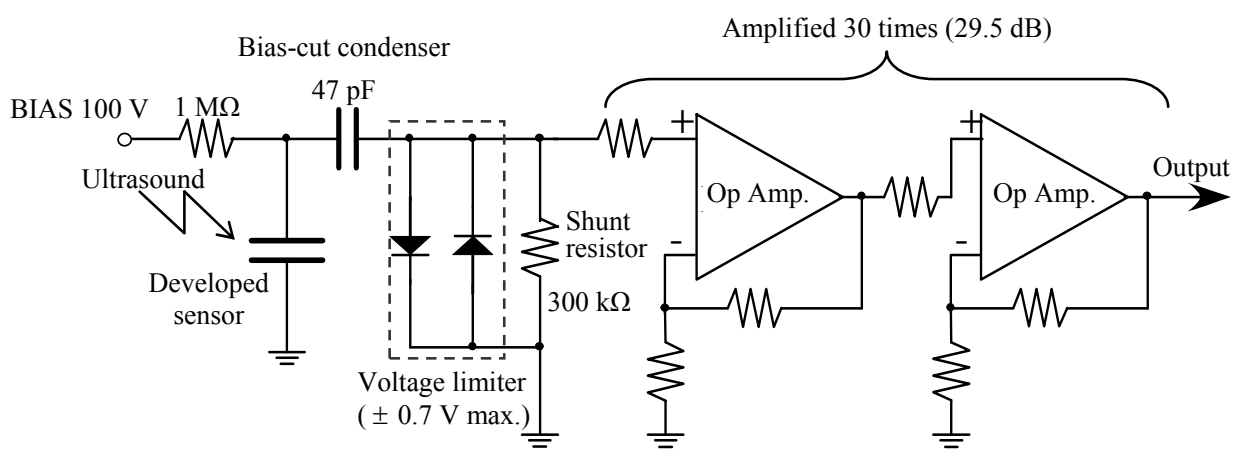

Fig. 15. CV transforming and amplifying circuit.

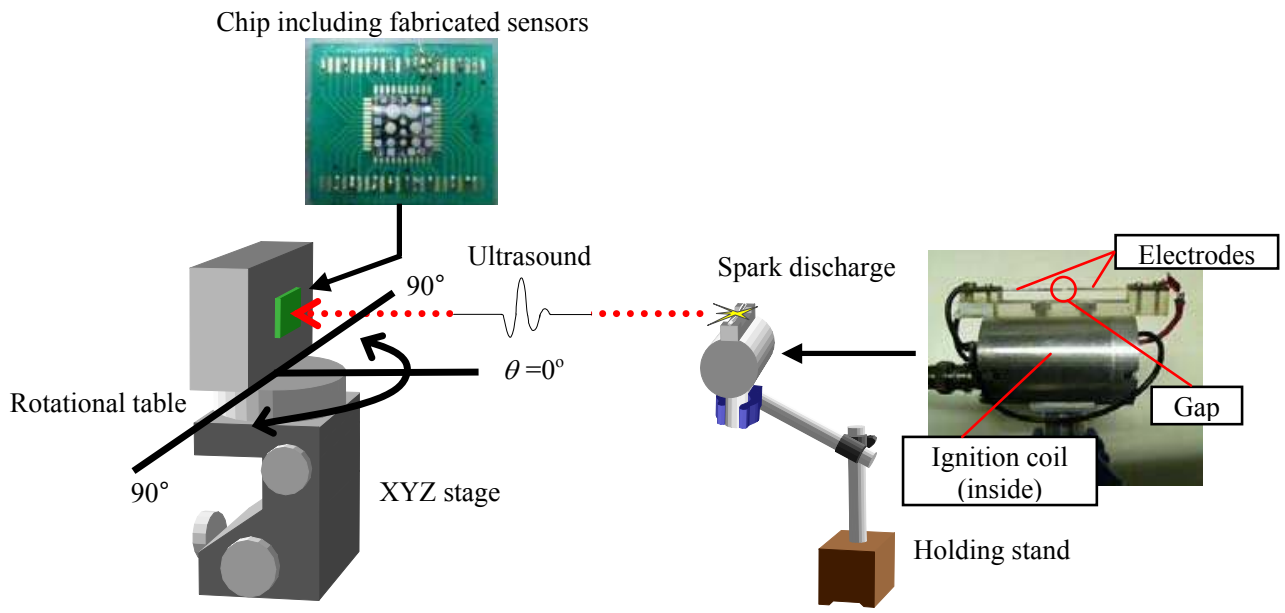

Fig. 16. Experimental condition for characterizing receiving performance.

Transmitted ultrasound is impulsive, the power spectrum of which is distributed over a broad frequency range (Aoyagi et al., 1992). The developed Parylene sensor was set on a rotational table. The distance between the transmitter and the sensor was set to $150 \mathrm{~mm}$. As a reference, a microphone to estimate the sound pressure at the same position where the sensor was set, B\&K type 4138 (already detailed in Section 1) was used.

\subsection{Received pulse waveform, sensitivity, and resonant frequency of one sensor}

An example of an ultrasonic pulse waveform received by the developed sensor, whose radius is $1,200 \mu \mathrm{m}$, is shown in Fig. 17. In this figure, the waveform received by the $B \& K$ microphone is also shown for reference. In the output signal of the developed sensor, there was electrical noise caused by the spark discharge, which could be suppressed by shielding the circuit completely in the future.

Considering that the sensitivity of the $\mathrm{B} \& \mathrm{~K}$ microphone is $0.9 \mathrm{mV} / \mathrm{Pa}$, and that the gain of amplification for the developed sensor is 30, the open-circuit sensitivity of the developed sensor was estimated to be $0.4 \mathrm{mV} / \mathrm{Pa}$. The value of typical commercial microphone is in the range from 1 to $50 \mathrm{mV} / \mathrm{Pa}$ for the audio range (Brüel \& Kjær, 1982; Knowles Acoustics, 


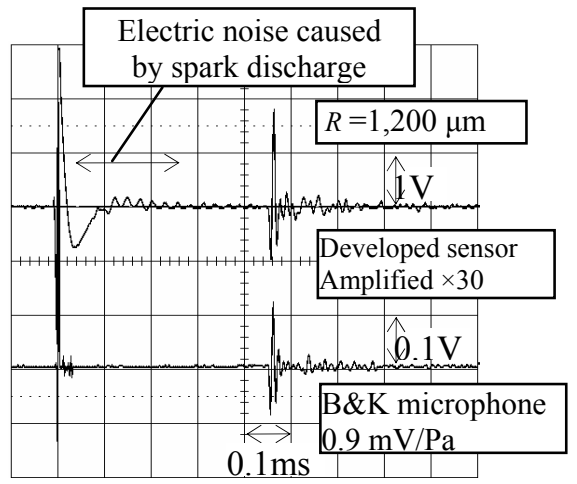

Fig. 17. Received ultrasonic waveforms by developed sensor and reference microphone.

2002). Considering that the diaphragm of the developed sensor is smaller than that of commercial microphone, the realized sensitivity is reasonable. In the end, the high sensitivity, the order of which is comparable with the B\&K microphone, was achieved.

In this study, the resonant frequency is defined as the reciprocal of the period between the first negative peak and the second one of the received waveform in a time domain, as shown in Fig. 18(a). An example of the power spectrum of the received waveform is shown in Fig. 18(b), which was obtained using a fast Fourier transform (FFT) analyzer. The resonant frequency measured based on the definition shown in Fig. 18(a) coincides well with the peak frequency in Fig. 18(b), which is $43 \mathrm{kHz}$ in the case of the sensor used. This value agrees well with FEM simulated value, as shown in Fig. 7, in which experimental data of resonant frequency of the developed sensors having different diaphragm sizes are plotted.

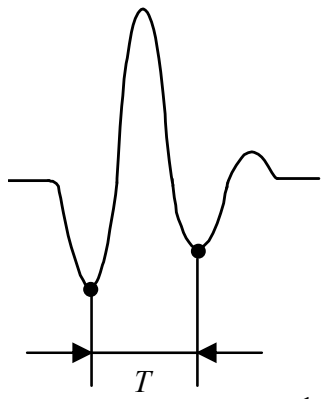

Resonant frequency $f_{r}=\frac{1}{T}$

(a) Definition of resonant frequency in time domain

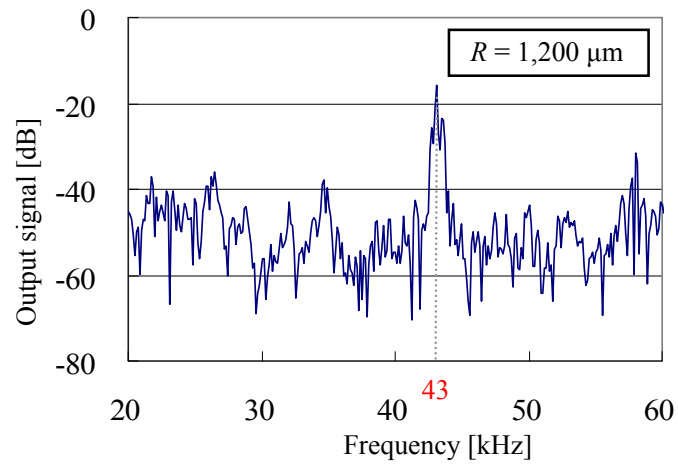

(b) Power spectrum of received waveform

Fig. 18. Measurement of resonant frequency.

\subsection{Fidelity for sound pressure and damping ratio}

The developed sensors with different sized acoustic holes, whose diaphragm radius is 1,200 $\mu \mathrm{m}$, were employed. The radius of an acoustic hole $(r)$ was 80,65 or $50 \mu \mathrm{m}$. The ultrasonic pulse waveforms received by the sensors are shown in Figs. 19(a)-(c). To estimate the 
fidelity, three waveforms for each sensor are shown. The waveform received by the B\&K microphone is also shown in Fig. 19(d) for reference.

The three waveforms in Fig. 19(a) resemble each other, as do those in Figs. 19(b) and (c). Thus, the reproducibility of the waveforms is good. In case that $r$ is $80 \mu \mathrm{m}$, the residual vibration of the waveform is seen, whereas there are no residual vibrations, i.e., the waveform is well damped, in case that $r$ is 65 and $50 \mu \mathrm{m}$. According to the FEM simulation results already shown in Fig. 9, the $\zeta$ values are 0.7, 1.0, and 1.1 for $r$ values of 80, 65, and $50 \mu \mathrm{m}$, respectively. When $\zeta$ exceeds 1.0 , there are no residual vibrations theoretically, which does not strongly contradict the experimental results, as shown in Figs. 19(b) and (c). The waveforms received by the developed sensors shown in Figs. 19(b) and (c) coincide well with that received by the B\&K microphone shown in Fig. 19(d), which confirms the high fidelity of the developed sensor for sound pressure in the ultrasonic frequency range, provided that an appropriate damping is given to it.

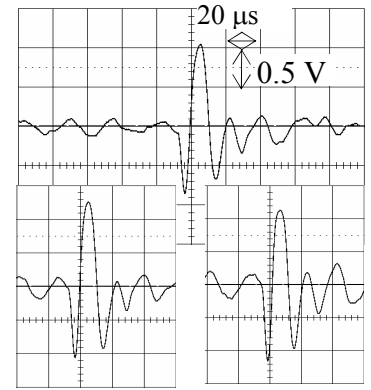

(a) $r=80 \mu \mathrm{m}$

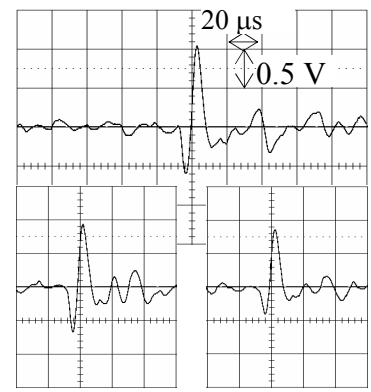

(c) $r=50 \mu \mathrm{m}$

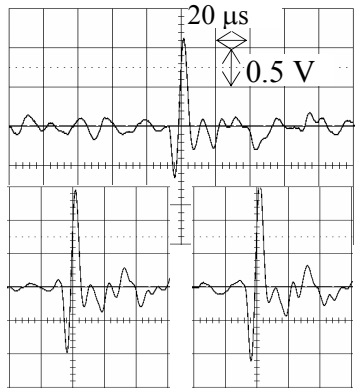

(b) $r=65 \mu \mathrm{m}$
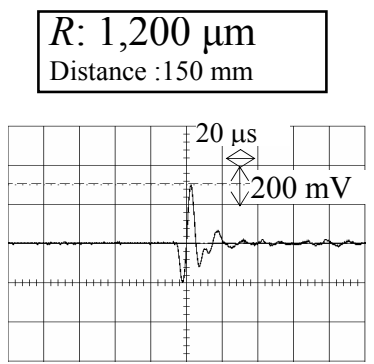

(d) B\&K microphone

Fig. 19. Received ultrasonic pulse waveforms by changing the radius $r$ of acoustic hole.

\subsection{Distance measurement}

The distance is measured by multiplying the arrival time of the first zero-cross point of the ultrasonic pulse by the sound velocity of $343.6 \mathrm{~m} / \mathrm{s}\left(\right.$ at $20^{\circ} \mathrm{C}$ ), as shown in Fig. 20. This point is stable and gives high resolution to the ranging system even when the amplitude varies according to the change in the distance. The sensor, whose diaphragm radius is $1,200 \mu \mathrm{m}$, was used. By changing the distance between the transmitter and the developed sensor, the arrival time was measured. The results for distance from 0 to 1,000 $\mathrm{mm}$ are shown in Fig. 21. 
The measured arrival time shows good linearity with the distance of the source, and error is within $0.1 \%$ of the full range, i.e., this ranging system can detect the distances up to $1 \mathrm{~m}$ with an error of less than $1 \mathrm{~mm}$. This ranging system could be effective for mobile robot devices for purposes such as detecting obstacles and recognizing the environment.

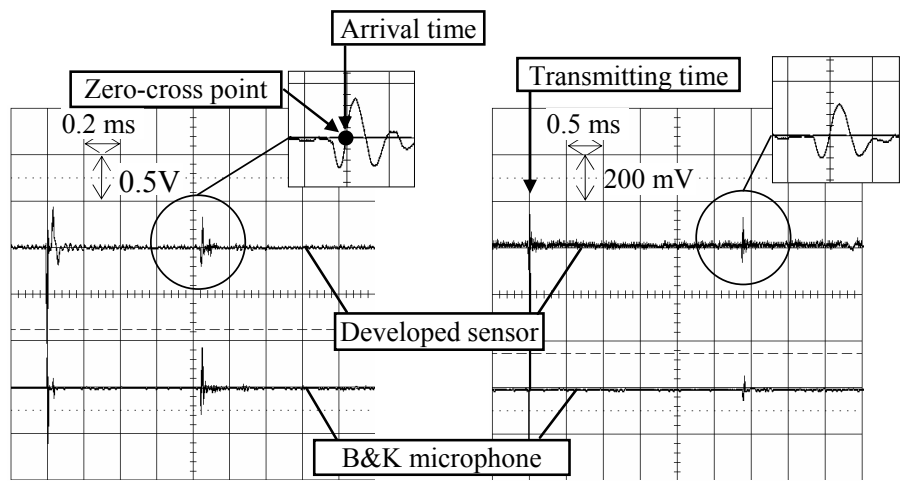

(a) Distance $=500$

(b) Distance $=1,000$

Fig. 20. Distance measurement by multiplying arrival time of zero-cross point by sound velocity.

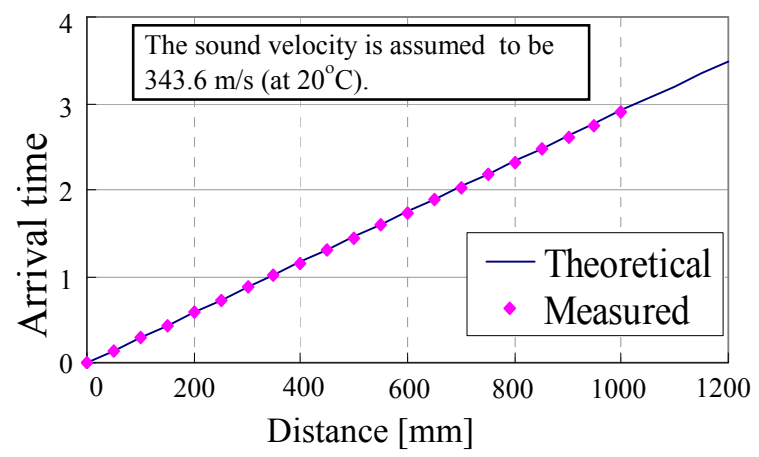

Fig. 21. Relationship between distance and measured arrival time.

\subsection{Receiving directivity of one sensor}

The directivity of the developed sensor was estimated using the experimental setup as already shown in Fig. 16. The peak voltage of received pulse waveform was estimated by changing the angle of the sensor using a rotational table. Results are shown in Fig. 22. From these results, the directivity becomes wide as the diaphragm radius decreases, which implies that miniaturizing the sensor size by micromachining is useful for achieving wide directivity.

It was confirmed that all the sensors used in this experiment can receive ultrasound from a wide area, which ranges from $\theta=-80$ to $80^{\circ}$, with an attenuation level of less than $-6 \mathrm{~dB}$ compared with the case $\theta=0^{\circ}$, i.e., $\theta_{1 / 2}$ (see equation (1) in Section 1) is approximately $80^{\circ}$. 
This wide directivity is effective for realizing the omnidirectional characteristics of the arrayed device comprising many sensors, the detail of which is explained in the following section.

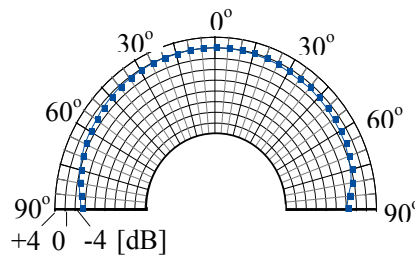

(a) $R=1,200 \mu \mathrm{m}$

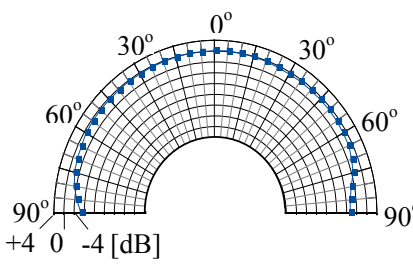

(c) $R=700 \mu \mathrm{m}$

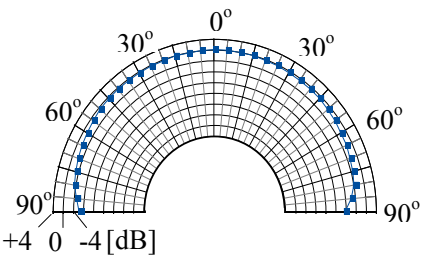

(b) $R=900 \mu \mathrm{m}$

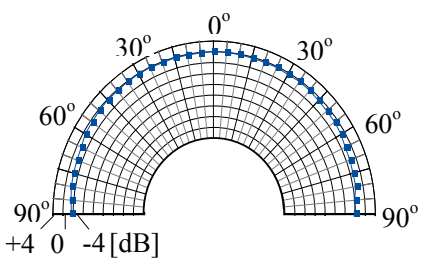

(d) $R=500 \mu \mathrm{m}$

Fig. 22. Receiving directivity of developed sensor.

\section{Arrayed sensor device and electrical scanning of receiving directivity}

\subsection{Detecting circuitry for capacitance change}

An arrayed device comprising $5 \times 5$ developed sensors was fabricated. A photograph and its actual size are shown in Fig. 23. The specification of one sensor in the array is as follows: the radius $(R)$ of the diaphragm is $1,200 \mu \mathrm{m}$, its thickness is $2 \mu \mathrm{m}$, the distance between adjacent diaphragms $(a)$ is $3,000 \mu \mathrm{m}$, the radius of the acoustic hole $(r)$ is $60 \mu \mathrm{m}$, and the number of holes $(n)$ is 121 .

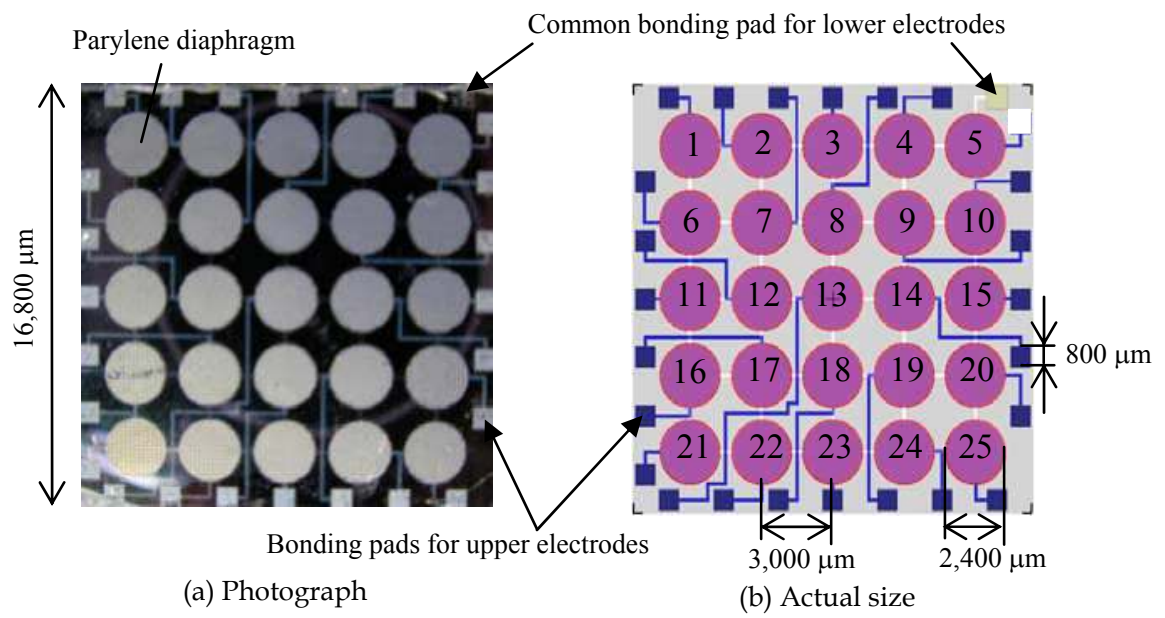

Fig. 23. Fabricated device of ultrasonic sensor array. 
The capacitance $(C)$, the dissipation factor $(\tan \delta)$, and the impedance $(Z)$ of individual sensors were measured using an LCZ meter (NF type 2341), examples of which are shown in Table 2. In this table, the wiring length for sensor no. 3 is the minimum and that for sensor no. 13 is the maximum among all the sensors, causing the difference of $C$ between them.

\begin{tabular}{cccc}
\hline Sensor no. & $\begin{array}{c}\text { Capacitance } \\
C[\mathrm{pF}]\end{array}$ & $\begin{array}{c}\text { Loss factor } \\
\tan \delta\end{array}$ & $\begin{array}{c}\text { Impedance at } 100 \mathrm{kHz} \\
Z[\mathrm{k} \Omega]\end{array}$ \\
\hline 3 & 36.0 & 0.02 & 41.9 \\
7 & 43.6 & 0.019 & 29.5 \\
13 & 69.5 & 0.024 & 19.5 \\
\hline
\end{tabular}

Table 2. Examples of electrical properties of one sensor.

\subsection{Dispersion of individual sensors' properties in arrayed device}

The distribution of sensitivity of individual sensors in the developed arrayed device was estimated, where the peak voltage of the received ultrasonic waveform is taken as the index of the sensitivity. The experimental results are shown in Fig. 24(a), the values of which do not strongly contradict the anticipated value of $67 \mathrm{mV}$ (see Section 4.3 and Fig. 17). There is dispersion of experimental sensitivity; however, it is not significant. Thus, the first zerocross point of the received pulse waveform can be detected in all the sensors by setting an appropriate threshold level, i.e., the time-of-flight measurement of ultrasound for determining the distance can be generally performed for all the sensors.

The distribution of the resonant frequency of individual sensors was also estimated. The experimental results are shown in Fig. 24(b), the values of which do not strongly contradict the target value of $43 \mathrm{kHz}$, which is confirmed by both FEM simulation (see Section 2.1 and Fig. 7) and experiments (see Section 4.3 and Fig. 18). However, the uniformity of resonant frequency is unsatisfactory.

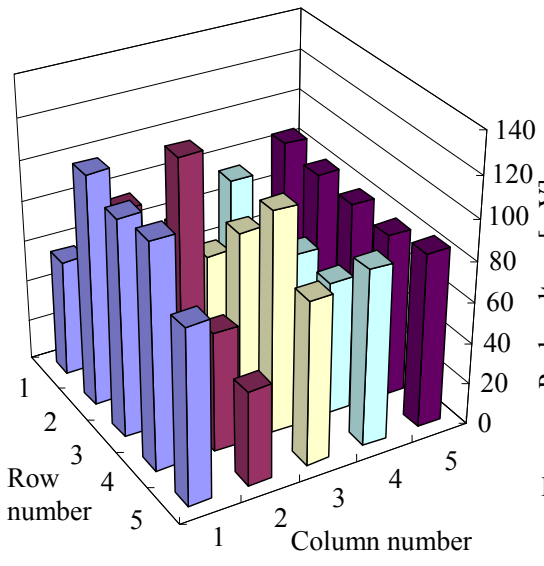

(a) Distribution of sensitivity

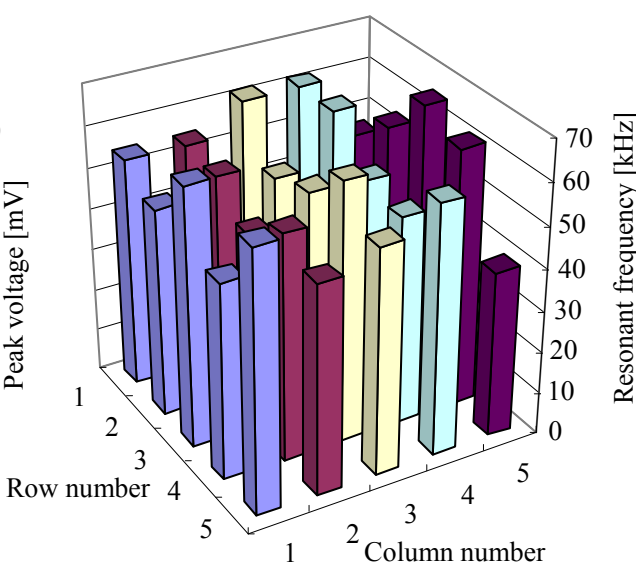

(b) Distribution of resonant frequencies

Fig. 24. Result of sensitivity and resonant frequency. 
One reason for the dispersion of resonant frequencies is due to the fabrication, i.e., the Young's modulus, thickness, and the intrinsic tensile stress of the Parylene diaphragm were not uniform all over the fabricated arrayed sensor area, since it is difficult to keep the process conditions strictly the same irrespective of the position inside the arrayed device. Because of this problem, the resonant frequency varied from one sensor to another, since the resonant frequency depends on these mechanical parameters (Khuri-Yakub et al., 2000; Aoyagi et al., 2007b). The process uniformity should be improved in future studies.

\subsection{Electrical scanning of receiving directivity}

The electric scanning of receiving directivity based on the delay-and-summation principle is possible by using many of sensors. Among totally twenty five sensors in the fabricated arrayed device, five sensors lying in one line were selected, and they were used for an experiment of performing the electrical scanning of receiving directivity, as shown in Fig. 25. The fabricated arrayed device was rotated using a rotational table, the center of which was set apart from an ultrasonic transmitter of electric spark discharge by $150 \mathrm{~mm}$. Let the rotational angle be $\theta$. Then the difference of sonic path length for two adjacent sensors is expressed as $a \sin \theta$, where $a$ is interval between the sensors ( $a=3,000 \mu \mathrm{m}$ in this case).

The procedure of the experiment is schematically shown in Fig. 26, which is as follows: Received pulse waveforms for the five sensors are schematically shown in Fig. 26(a). Their arrival times have differences based on the differences in sonic path length. After recording the waveforms in a computer, the positive peak of each waveform is detected. Taking this peak as the center, a rectangular pulse wave with $5 \mu$ s width is generated, as shown in Fig. 26(a). Then, each pulse is shifted by a delay time of $\{(n-1) \cdot a \sin \alpha\} / v$, where $\alpha$ is the scanning angle of directivity, $v$ is the sound velocity $(343.6 \mathrm{~m} / \mathrm{s}$ is employed in this experiment), and $n$ is the number of the sensor which takes $1,2, \cdots, 5$. The shifted pulses are summed, and the area inside the width of pulse no. 1 is extracted from the summed result, which is the hatched area shown in Fig. 26(b). The average height of this area is estimated as the index of sensitivity.

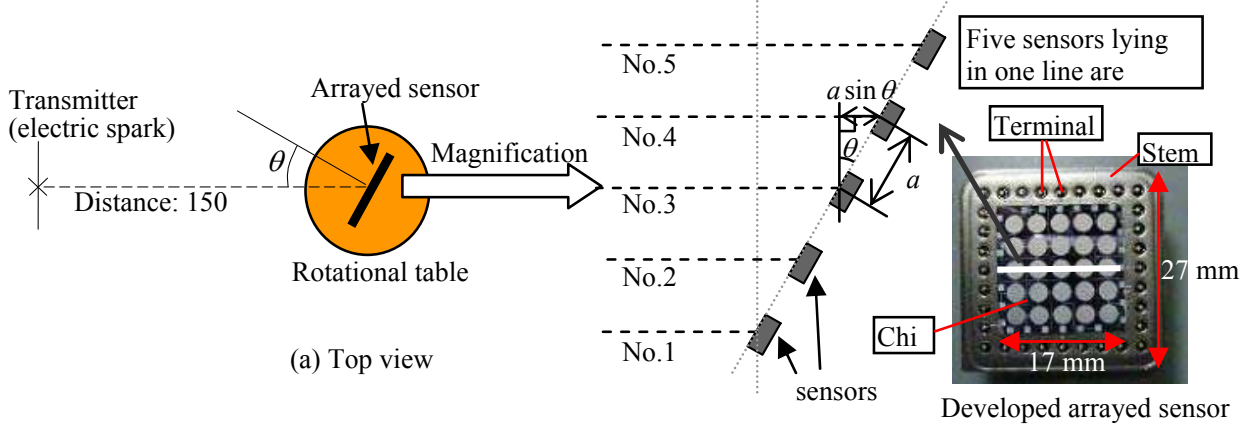

(b) Schematic magnification of five

Fig. 25. Experimental conditions for electrical scanning of receiving directivity using arrayed sensor device. 
$\alpha$ : scanning angle of directivity, $v$ : sound velocity

$\theta:$ true angle of direction of the transmitter

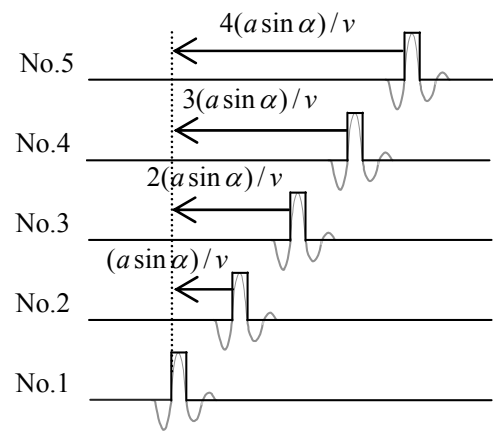

(a) Peak is detected, and rectangular wave with 5 $\mu$ s width is generated. Each pulse is shifted by delay time and summed up.

[V]

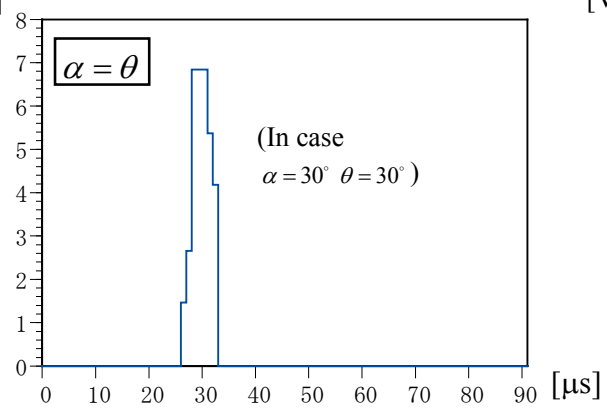

(b) The area inside the width of pulse no. 1 is obtained and estimated.
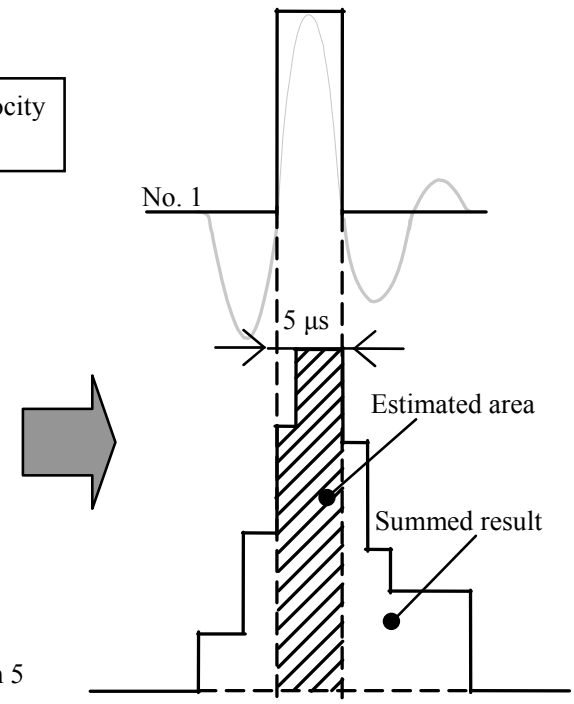

]

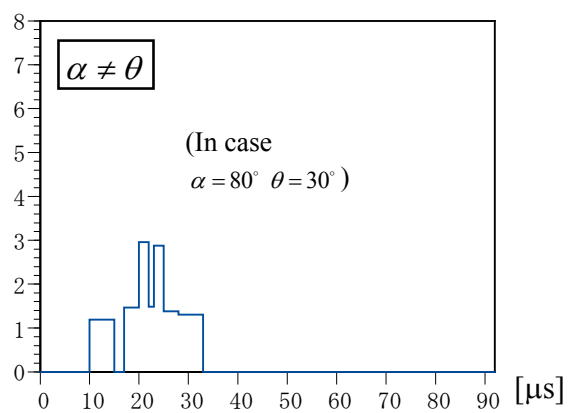

(c) Examples of actual summed rectangular waveforms

Fig. 26. Procedure of electrical scanning of receiving directivity using arrayed sensor.

Examples of actual summed rectangular waveforms are shown in Fig. 26(c). Looking at this figure, the width of the summed result almost coincides with that of pulse no. 1, i.e., it almost fits inside a $5 \mu$ s width in the case of $\alpha=\theta$, while it does not do so in the case of $\alpha \neq \theta$. Namely, the sensitivity is maximized in the former case.

These processes, i.e., detecting peaks, generating pulses, shifting them, summing them, and extracting the area for estimation, were performed by developed computer software. In the experiment, $\theta$ was set at $0,10, \cdots, 90^{\circ}$. For each $\theta$, a scanning angle $\alpha$ of $0,10, \cdots 90^{\circ}$ was tested computationally, and the sensitivity of each combination of $\theta$ and $\alpha$ was estimated. 
The results of electrical scanning performance of receiving directivity are shown in Fig. 27. In this figure, each data is normalized to a relative value in $\mathrm{dB}$ units, so that the sensitivity when $\theta=\alpha$ is $0 \mathrm{~dB}$. The absolute value of the sound pressure level (SPL) for the case of $0 \mathrm{~dB}$ for each $\theta$ angle is shown in Table 3. Looking at this table, the SPL does not decrease as $\theta$ increases, i.e., it takes almost the same value irrespective of $\theta$.

According to Fig. 27, the sensitivity is increased when $\alpha=\theta$, i.e., when the scanning angle $(\alpha)$ is coincident with the angle of direction of the transmitter $(\theta)$, except for only the two cases of $\theta=70$ and $80^{\circ}$. Even in these two cases, the error is small, within $10^{\circ}$. Note that when $\theta$ is in the range from 0 to $50^{\circ}$, a sharp peak of directivity at the target scanning angle is obtained, which may be effective for detecting an angle at which a target object exists in microrobot applications. To conclude, it was proven that the directivity can be scanned electrically based on the delay-and-summation principle using the fabricated Parylene arrayed device. It was also proven that a wide scanning angle of at least $50^{\circ}$ can be achieved. This omnidirectional characteristic is due to the wide directivity of the individual sensor, which was already characterized in Section 4.6.

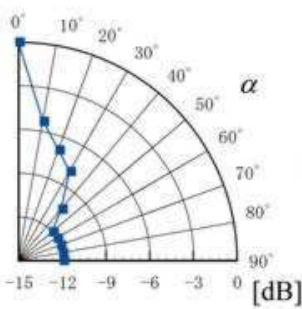

(a) $\theta=0^{\circ}$

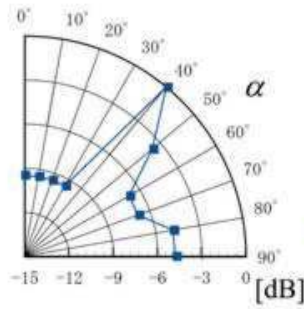

(e) $\theta=40^{\circ}$

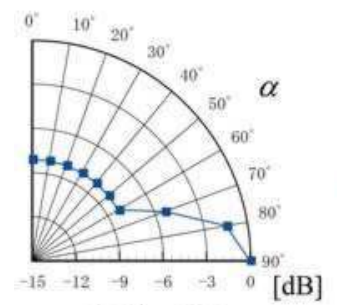

(i) $\theta=80^{\circ}$

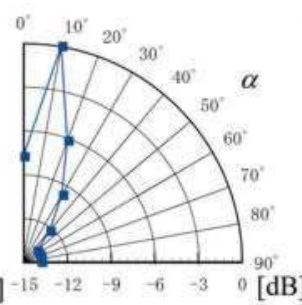

(b) $\theta=10^{\circ}$

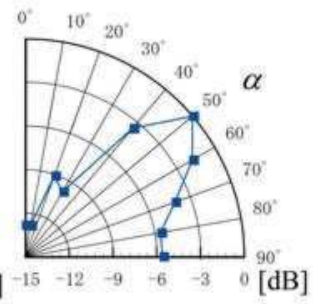

(f) $\theta=50^{\circ}$

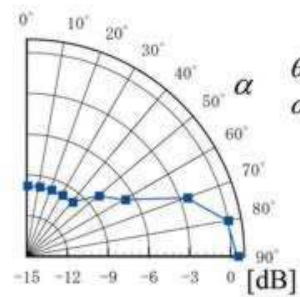

(j) $\theta=90^{\circ}$

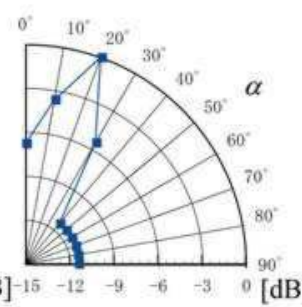

(c) $\theta=20^{\circ}$

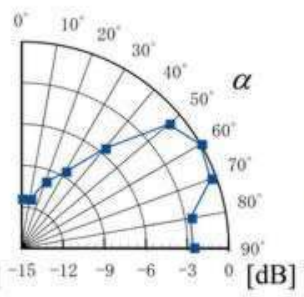

(g) $\theta=60^{\circ}$

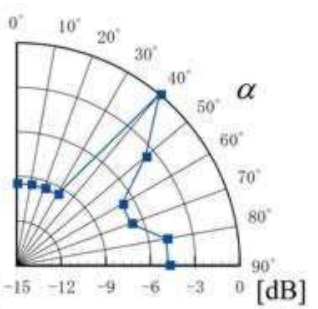

(d) $\theta=30^{\circ}$

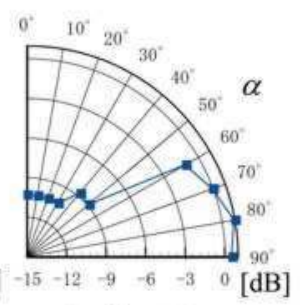

(h) $\theta=70^{\circ}$

$\theta:$ True anagle of direction, in which the transmitter exists. $\alpha$ : Scanned angle of directivity.

Each data is normalized, so as that the sensitivity when $\alpha=\theta$ is to be $0[\mathrm{~dB}]$.

It is shown that the sensitivity from the $\theta$ direction is intensified by setting a delay time of $t=(a \sin \theta) / v$.

Fig. 27. Results of electric scanning of receiving directivity using arrayed sensor. 


\begin{tabular}{cc}
\hline$\theta\left[{ }^{\circ}\right]$ & SPL $[\mathrm{dB}]$ \\
\hline 0 & 152 \\
10 & 150 \\
20 & 145 \\
30 & 148 \\
40 & 142 \\
50 & 147 \\
60 & 145 \\
70 & 141 \\
80 & 140 \\
\hline
\end{tabular}

Table 3. Sound pressure level (SPL) for $0 \mathrm{~dB}$ case in Fig. 27 for each $\theta$.

\section{Transmitting performance of one sensor and electrical scanning of transmitting directivity}

\subsection{Transmitting circuitry}

Because of the flexibility and durability of Parylene, one capacitive sensor with a Parylene diaphragm can also be used as a transmitter by applying a high impulsive voltage. A transmitting circuit was developed, as shown in Fig. 28(a), in which the same bias voltage of $100 \mathrm{~V}$ as that used in the receiving circuitry is employed. When the transistor is triggered, a condenser $C_{T}$ of $0.1 \mu \mathrm{F}$ is discharged and an electric current is instantaneously supplied to the primary side of the ignition coil. Then a high impulsive voltage is generated at the secondary side of this coil, as shown in Fig. 28(b), which exhibits a peak-to-peak voltage of approximately $700 \mathrm{Vpp}$ (the positive voltage of $400 \mathrm{Vop}$ and negative one of $300 \mathrm{Vop}$, both of which are values relative to the bias voltage of $100 \mathrm{~V})$. The power spectrum of this voltage is shown in Fig. 28(c). In this figure, the peak frequency is $310 \mathrm{kHz}$, which is far larger than the resonant frequency of the developed device $(43 \mathrm{kHz})$. This fact indicates that the response of the diaphragm's displacement at the transmission can be approximately regarded as an impulse response, on which the resonant frequency of the diaphragm has a large effect rather than the peak frequency of the input voltage.

\subsection{Experimental setup for characterizing transmitting performance}

The transmitting performance of the developed Parylene device was characterized. The experimental setup is schematically shown in Fig. 29. The device was set on a rotational table. Each sensor in the arrayed device was activated as a transmitter. In addition to the arrayed device, a device including several sensor/transmitters with different radii of the diaphragm and different radii of the acoustic hole was prepared. This device was used to investigate the effect of the area of the diaphragm on the transmitted sound pressure and the effect of the acoustic holes on damping of the transmitted waveform.

The B\&K-type 4138 reference microphone (with sensitivity $0.9 \mathrm{mV} / \mathrm{Pa}$ ) was used as a receiver. The distance between the center of the arrayed transmitter device and the receiver was set to several values ranging from 10 to $1,000 \mathrm{~mm}$ to characterize the performance of one transmitter, and $40 \mathrm{~mm}$ to perform the electrical scanning of the arrayed transmitter. In the case that the transmitted acoustic pressure is small, the received signal obtained by the reference microphone was amplified by a factor of 3,000 (69.5 dB) using an instrumentation amplifier (ACO type 6030). 


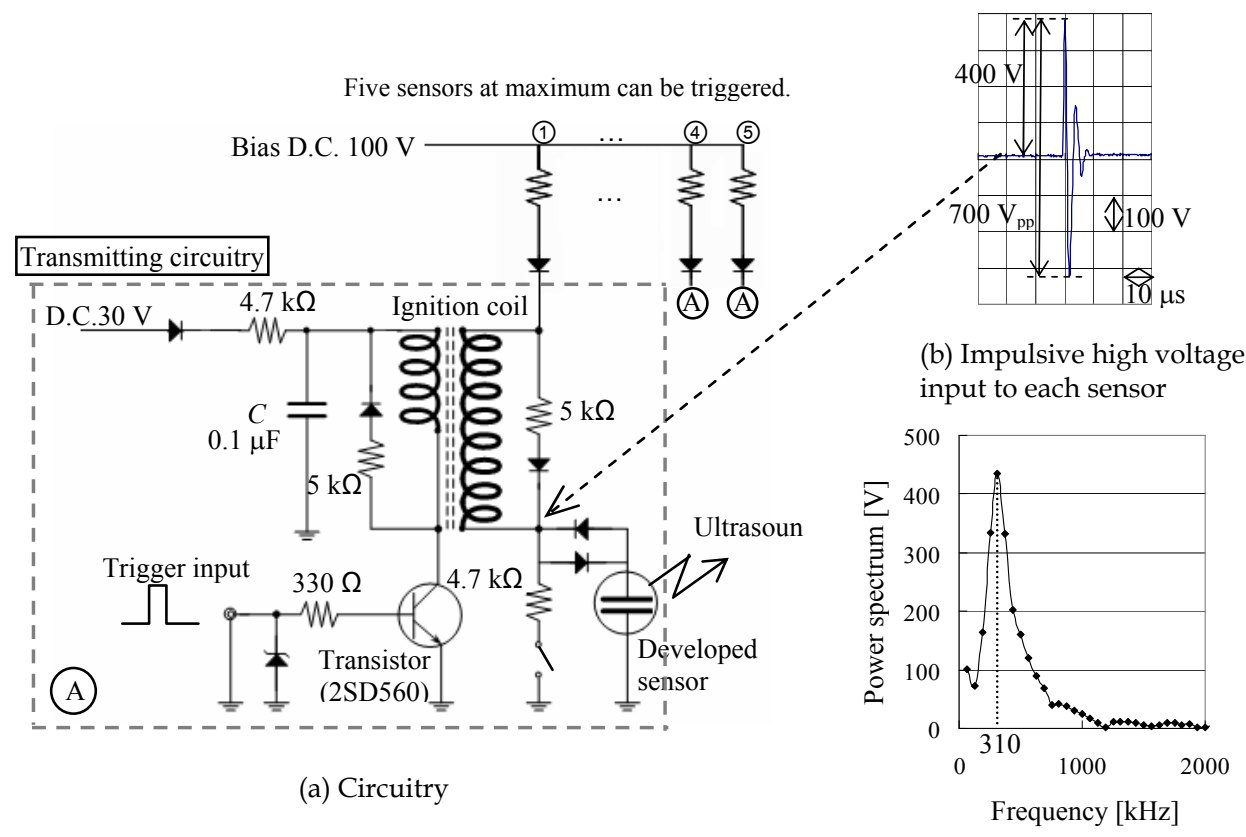

(c) Its power spectrum

Fig. 28. Transmitting circuitry of generating a high impulsive voltage.

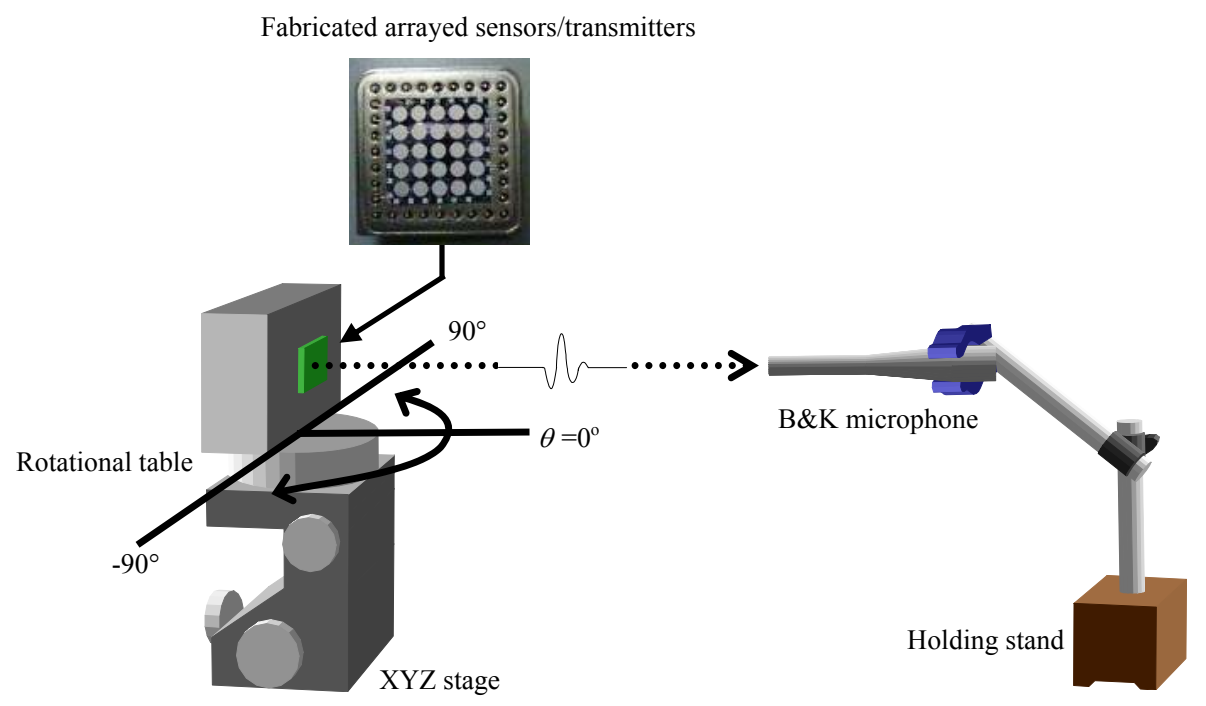

Fig. 29. Experimental conditions for characterizing transmitting performance. 


\subsection{Transmitted pulse waveform and detectable distance}

The ultrasonic waveform, which is emitted by the developed transmitter and received by the B\&K-type 4138 reference microphone, is shown in Fig. 30(a). The acoustic pressure obtained at a distance of $10 \mathrm{~mm}$ was $13 \mathrm{~Pa}$, which is rather small. Therefore, the signal was amplified using an instrumentation amplifier. The amplified received waveform obtained at a distance of $150 \mathrm{~mm}$ is shown in Fig. 30(b). By this amplification, the maximum distance at which the transmitted waveform is detectable was extended. The experimental results of the relationship between the distance and the peak voltage of the transmitted waveform are shown in Table 4, which indicates that the transmitted waveform can be detected as far as $1,000 \mathrm{~mm}$ away by setting an appropriate threshold level. It was confirmed that the developed transmitter is useful for the application of ranging the distance based on the time-of-flight measurement in the air.

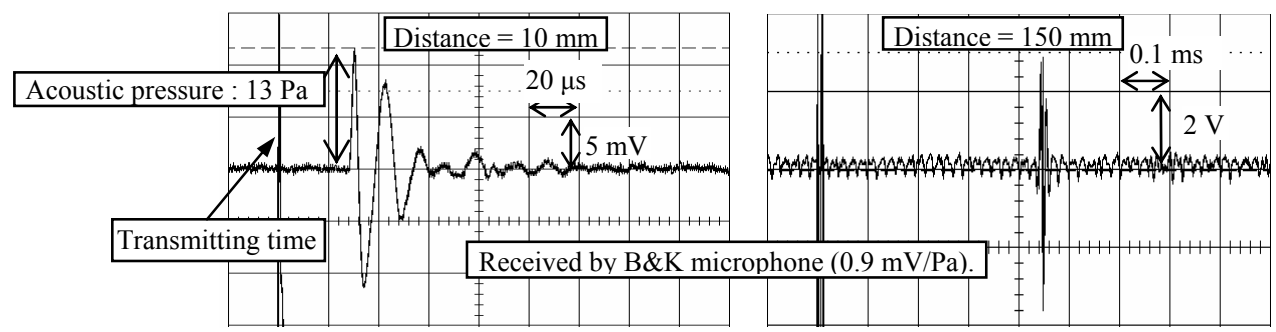

(a) Without amplification

(b) Amplified by 3,000 times $(69.5 \mathrm{~dB})$ by instrumentation amplifier.

Fig. 30. Emitted waveforms by developed transmitter $(R=1,200 \mu \mathrm{m})$.

\begin{tabular}{ccccc}
\hline Distance [mm] & 100 & 300 & 600 & 1,000 \\
Peak voltage [V] & 2.2 & 1 & 0.8 & 0.4 \\
\hline
\end{tabular}

Note: B\&K microphone output was amplified by $69.5 \mathrm{~dB}$ and estimated.

Table 4. Relationship between distance and peak voltage of transmitted waveform.

\subsection{Effect of diaphragm area on transmitted sound pressure}

The pulse waveforms emitted by the developed transmitters, of which the diaphragm radii are $500,700,900$, and $1,200 \mu \mathrm{m}$, were obtained, and their peak voltages were transformed to the sound pressure. The relationship between the diaphragm area and the transmitted sound pressure at $150 \mathrm{~mm}$ distance is shown in Fig. 31. It was proven that the sound pressure increases proportionally with the diaphragm area.

\subsection{Effect of acoustic holes on damping of transmitted waveform}

We have theoretically investigated the effects of the radius of the acoustic hole $r$ and the number of holes $n$ on the diaphragm's damping ratio $\zeta$ in Section 2.2. It was proven that $\zeta$ is inversely proportional to $r$ and $n$, which was also experimentally confirmed by the ultrasonic waveform received by the developed sensor as explained in Section 4.4. In this section, we aim to confirm this effect of acoustic holes by the ultrasonic waveform emitted by the developed transmitter. 


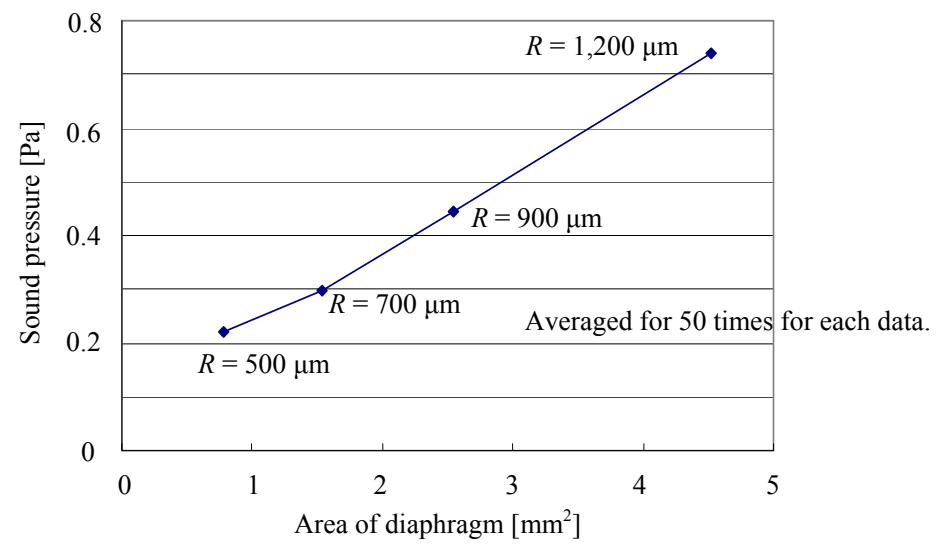

Fig. 31. Relationship between area of diaphragm and transmitted sound pressure.

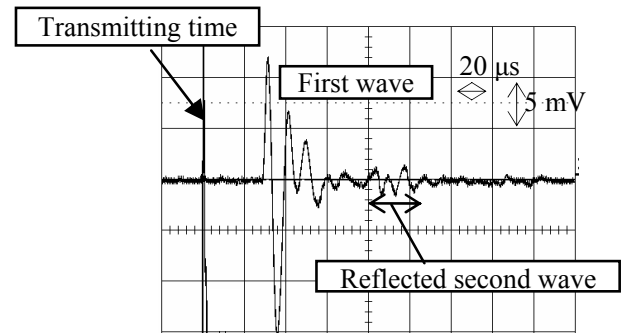

(a) $r=80 \mu \mathrm{m}$

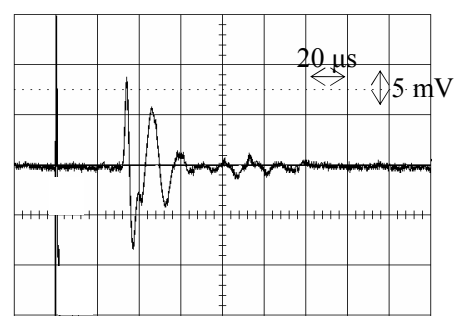

(c) $r=55 \mu \mathrm{m}$

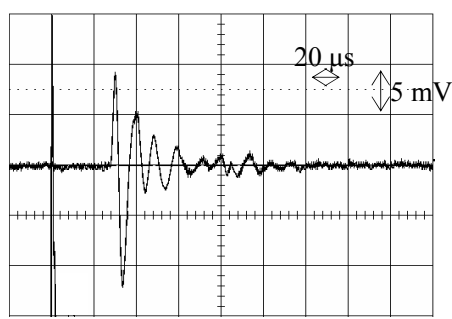

(b) $r=75 \mu \mathrm{m}$

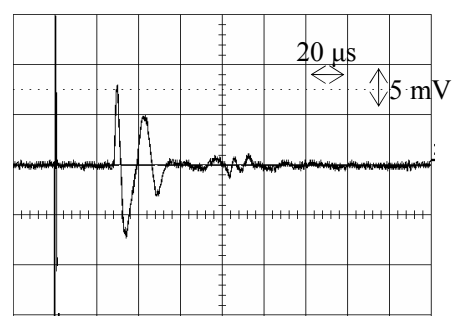

(d) $r=50 \mu \mathrm{m}$

Fig. 32. Transmitted waveforms at distance of $10 \mathrm{~mm}$ by changing the radius $r$ of acoustic hole.

The developed transmitters with different sizes of acoustic holes, of which diaphragm radius is $1,200 \mu \mathrm{m}$, were employed. The radii of the acoustic holes $r$ are 80, 75, 55, and 50 $\mu \mathrm{m}$. The ultrasonic pulse waveforms emitted are shown in Figs. 32(a)- (d). The distance was set to $10 \mathrm{~mm}$, and the waveform was detected by the $B \& \mathrm{~K}$ microphone with no amplification. Note that a second small waveform is also observed in this figure, which is reflected by the $B \& K$ microphone, returns to the transmitter, reflected by the transmitter, and again returns to the microphone. 
According to this figure, a well-damped transmitted waveform is obtained when $r$ is 55 or $50 \mu \mathrm{m}$, whereas a residual vibration is seen when $r$ is 80 or $75 \mu \mathrm{m}$. Namely, it was confirmed that $\zeta$ is inversely proportional to $r$. The effect of acoustic holes on the diaphragm damping confirmed here using the transmitted waveform does not contradict that confirmed using the received waveform.

\subsection{Directivity of one transmitter}

The directivity of the developed transmitter was estimated using the experimental setup shown in Fig. 29. The distance between the transmitter and the sensor was set to $150 \mathrm{~mm}$, and the peak voltage of the received pulse waveform was estimated by changing the angle of the transmitter using a rotational table. Results are shown in Fig. 33. From these results, the directivity becomes wide as the diaphragm radius decreases. It was confirmed that both of the transmitters used in this experiment can emit ultrasound over a wide direction, which ranges from $\theta=-80$ to $80^{\circ}$, with an attenuation level of less than $-4 \mathrm{~dB}$ compared with the case where $\theta=0^{\circ}$. Namely, the developed transmitter can be approximated to be nondirectional.

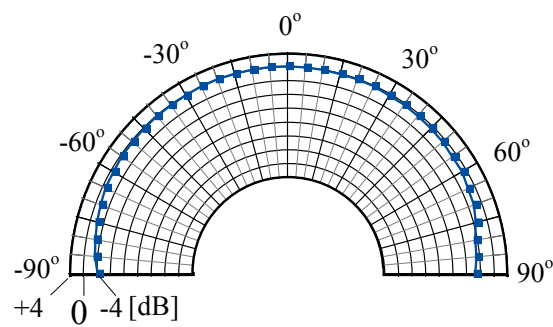

(a) $R=1,200 \mu \mathrm{m}$

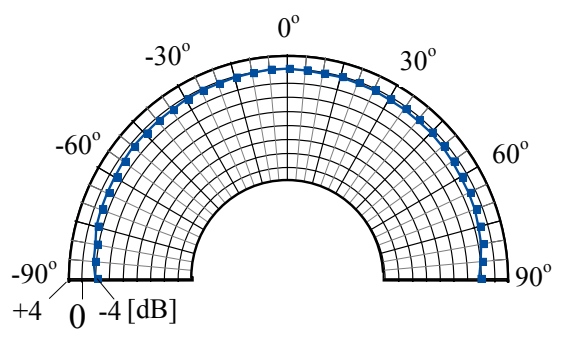

(b) $R=900 \mu \mathrm{m}$

Fig. 33. Transmitting directivity of developed transmitter.

\subsection{Electrical scanning of transmitting directivity}

Five collinear transmitters were selected, and they were used for an experiment of performing the electrical scanning of transmitting directivity. The experimental conditions are schematically shown in Fig. 34(a). The fabricated arrayed device was rotated using a rotational table. Let the rotational angle be $\theta$. Then the difference of the sonic path length for two adjacent transmitters is expressed as $a \sin \theta$, where $a$ is interval between the transmitters. The procedure, based on the delay-and-summation principle, is as follows. Trigger input pulses for the five transmitters are schematically shown in Fig. 34(b). When the frequency of these pulses is set to $f=v /(a \sin \alpha)$, the transmitted waves are theoretically intensified in the $\alpha$ direction, where $\alpha$ is the scanning angle of directivity, and $v$ is the sound velocity ( $343.6 \mathrm{~m} / \mathrm{s}$ is employed in this experiment).

For each $\theta$, the scanning angle $(\alpha)$ was set by changing the frequency $(f)$ of the trigger pulses, which were input to the transmitting circuitry. The peak voltage of transmitted waveform, which is received by the B\&K microphone, was estimated at each combination of $\theta$ and $f$. 


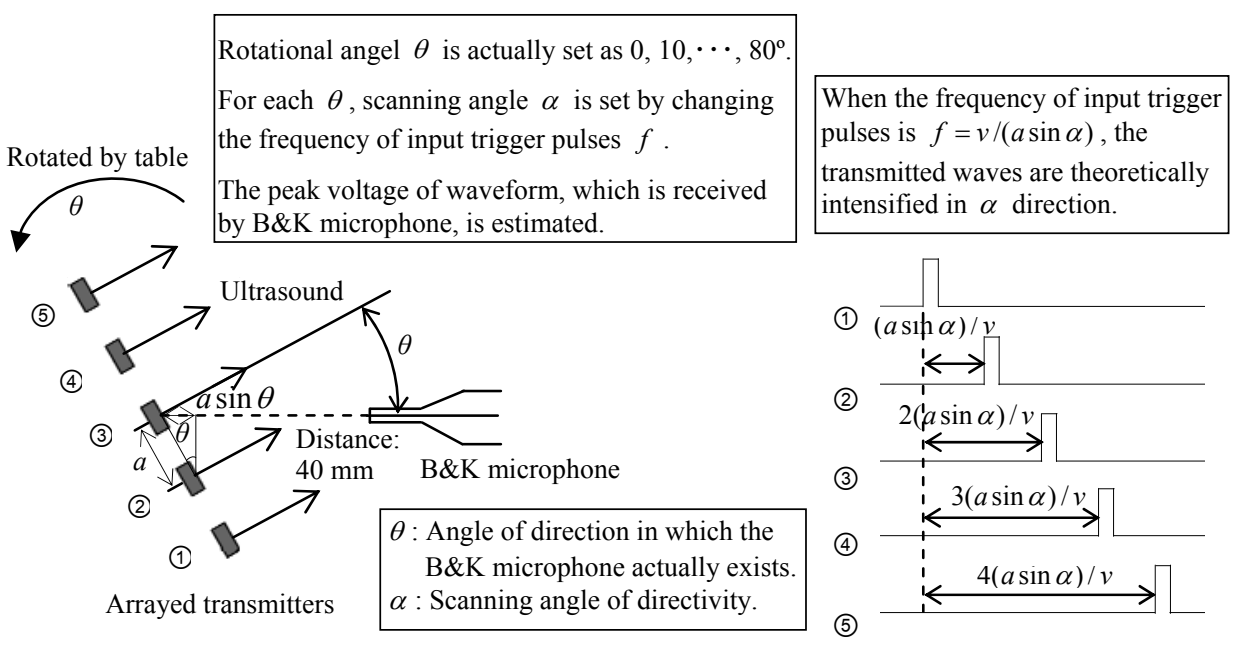

(a) Schematic of experimental setup using rotational table and reference microphone (B \& K 4138) (b) Shifting trigger inputs to each sensor for controlling the transmitting directivity

Fig. 34. Experimental conditions for electrical scanning of transmitting directivity using arrayed device.

The results of electrical scanning performance of transmitting directivity are shown in Fig. 35. In this figure, each data is normalized, so that the detected peak voltage when $f=v /(a \sin \theta)$, i.e., $\alpha=\theta$, is $0 \mathrm{~dB}$. According to this figure, the transmitted waveform was intensified at $f=v /(a \sin \theta)$, i.e., it was intensified when the scanning angle $(\alpha)$ was coincident with the angle of the direction $(\theta)$ of the receiver. However, the directivity when $\theta=30^{\circ}$ was less sharp than that in the other conditions in this figure. This may be caused by an experimental problem, the improvement of which is a possible future study. To conclude, although further study is necessary, the possibility of controlling the transmitting directivity was preliminarily shown in this experiment using the fabricated arrayed device.

\section{Conclusions}

An arrayed device comprising $5 \times 5$ ultrasonic sensors/transmitters featuring polymer Parylene diaphragms was fabricated, and its performance was characterized. In addition to the durability and high sensitivity due to polymer nonbrittleness and flexibility, merits attributable to Parylene, such as biocompatibility, chemical resistivity, CMOS compatibility, and conformal deposition, are expected to be achieved in the future.

The contents of this study are briefly summarized as follows. 1) An ultrasonic sensor with Parylene diaphragm was developed. The sensor was found to be able to receive an impulsive ultrasonic pulse transmitted by a spark discharge. The open-circuit sensitivity was $0.4 \mathrm{mV} / \mathrm{Pa}$. 2) A well-damped waveform was obtained by setting appropriate acoustic 


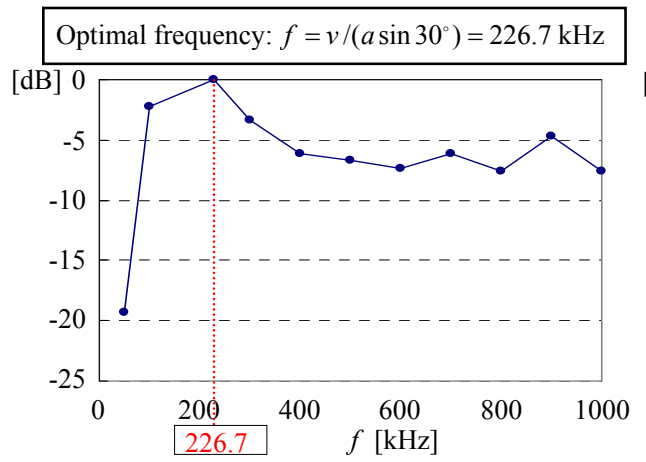

(a) $\theta=30^{\circ}$

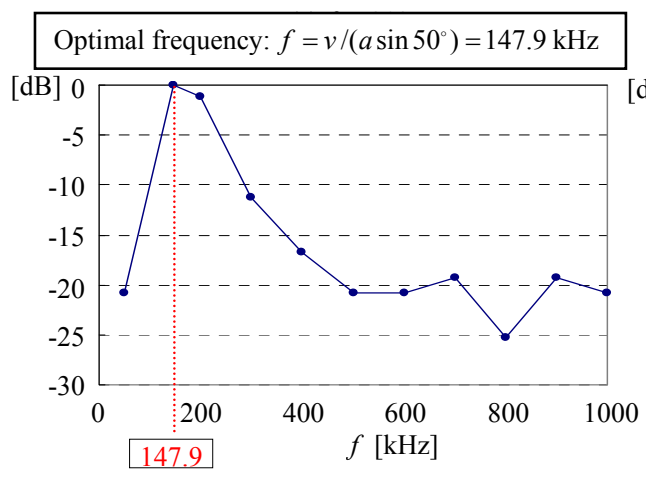

(c) $\theta=50^{\circ}$

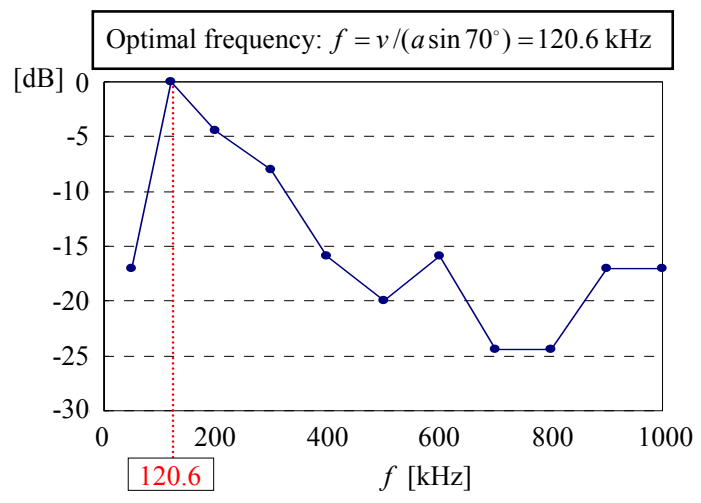

(e) $\theta=70^{\circ}$
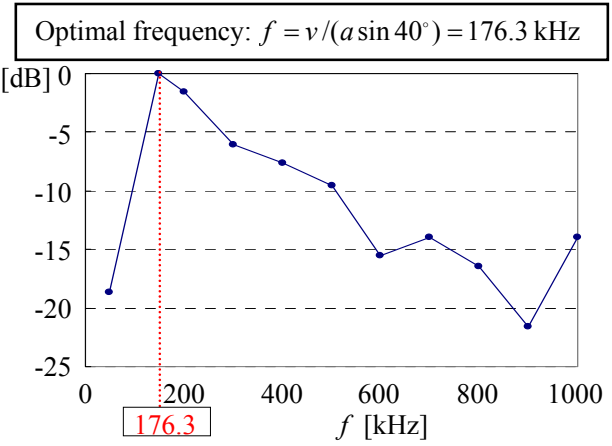

(b) $\theta=40^{\circ}$
Optimal frequency: $f=v /\left(a \sin 60^{\circ}\right)=130.9 \mathrm{kHz}$

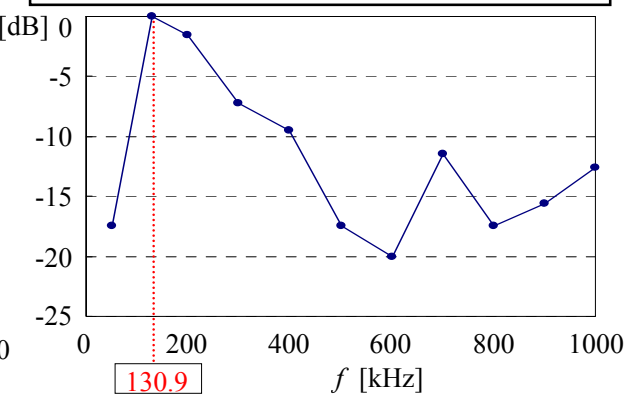

(d) $\theta=60^{\circ}$
Each data is normalized, so that the peak value when $f=v / a \sin \theta$ is $0 \mathrm{~dB}$.

Fig. 35. Results of electric scanning of transmitting directivity using arrayed device. 
holes. 3) The ranging system using this sensor can detect distances up to $1 \mathrm{~m}$ with an error of less than $1 \mathrm{~mm}$. 4) The developed sensor can receive ultrasound from a wide area, which ranges from $\theta=-80$ to $80^{\circ}$. 5) An arrayed ultrasonic device was developed by the micromachining technique. The dispersion of individual sensors' properties, i.e., the sensitivity and the resonant frequency, was proven to be tolerable. 6) The electrical scanning of receiving directivity was performed on the basis of the delay-and-summation principle. A wide scanning angle of at least $50^{\circ}$ was achieved. 7) Each developed sensor was activated as a transmitter by applying a high impulsive voltage. The transmitted waveform was detectable as far as $1,000 \mathrm{~mm}$ away. The ultrasound was transmitted over a wide direction ranging from $\theta=-80$ to $80^{\circ}$. 8) The possibility of electrical scanning of transmitting directivity was preliminarily confirmed using the developed arrayed device.

By scanning both the transmitting directivity and the receiving directivity of the developed arrayed device, detecting the direction in which objects or obstacles exist is a future study. In this study, by detecting the time-of-flight of an ultrasonic pulse reflected by objects or obstacles, the distance from them is also detectable. By using the information on both the direction and the distance, the positions of objects or obstacles may be obtained in the future. Further quantitative investigation of the merits of the developed Parylene ultrasonic arrayed sensors/transmitters compared with other reported silicon or polymer devices is also a planned future study.

\section{Acknowledgements}

This work was supported by a Grant-in-Aid for Scientific Research (17656090) from the Ministry of Education, Culture, Sports, Science and Technology (MEXT) and the "High-Tech Research Center" Project for Private Universities: Matching Fund Subsidy from MEXT, 20052009, and the Kansai University Special Research Fund, 2007 and 2008. The author is grateful to Professors Hiromitsu Kozuka and Yasuhiko Arai at Kansai University for their invaluable advice and for supplying measuring apparatus. The author is grateful to Mr. Go Kawai for his drawing figures.

\section{References}

Aoyagi, S.; Kamiya, Y. \& Okabe, S. (1992). Development of Powerful Airborne Ultrasonic Transmitter for Robot Metrology. Proc. the 12th Symposium on Ultrasonic Electronics, Japanese J. Applied Physics, Vol. 31, Suppl. 31-1, pp. 263-265.

Aoyagi, S. (1996). Application of Ultrasonic Sensors to Robot Measurement. J. the Japan Society for Precision Engineering, Vol. 62, No. 3, pp. 373-376 (in Japanese).

Aoyagi, S. \& Takehata, K. (2001). Study on Object Shape Recognition Using an Ultrasonic Sensor. Integrated Computer-Aided Engineering, Vol. 8, pp. 105-117.

Aoyagi, S.; Furukawa, K.; Yamashita, K.; Tanaka, T.; Inoue, K. \& Okuyama, M. (2007a). Development of Capacitive Ultrasonic Sensor with Parylene Diaphragm Using Micromachining Technique. Japanese J. Applied Physics, Vol. 46, pp. 4595-4601.

Aoyagi, S.; Yoshikawa, D; Isono, Y \& Tai,Y,C. (2007b). Development of a Capacitive Accelerometer Using Parylene (Part 1) -Study on Resonant Frequency of Parylene Suspended Structure-, IEEJ Trans. SM, Vol.127, No.6, pp. 314-320. 
Aoyagi, S; Furukawa, K; Ono, D; Yamashita, K; Tanaka, T; Inoue, K \& Okuyama, M. (2008a). Development of a capacitive ultrasonic sensor having parylene diaphragm and characterization of receiving performance of arrayed device. Sensors and Actuators A, Vol. 145-146, pp. 94-102.

Aoyagi, S; Ono, D; Kawai, G; Yamashita, K; Okuyama, M. (2008b). Micromachined Arrayed Capacitive Ultrasonic Sensor/Transmitter with Parylene Diaphragms, Japanese J. Applied Physics, Vol. 47, No. 8, pp. 6513-6525.

Bergqvist, J. \& Gobet, J. (1994). Capacitive Microphone with a Surface Micromachined Backplate Using Electroplating Technology. J. Microelectromechanical Systems, Vol. 3, No. 2, pp. 69-75.

Brüel \& Kjær (1982). Condenser Microphones Data Handbook, Brüel \& Kjær, Nærum, Denmark.

Chen, J.; Liu, L.; Li, Z.; Tan, Z.; Xu, Y. \& Ma, J. (2002). Single-Chip Condenser Miniature Microphone with a High Sensitive Circular Corrugated Diaphragm. Proc. MEMS'02, pp. 284-287, Las Vegas, USA, January, 2002.

Diamond, B, M.; Neumann, J, J. \& Gabriel, K, J. (2002). Digital Sound Reconstruction Using Arrays of CMOS-MEMS Microspeakers. Proc. MEMS'02, pp.292- 295, Las Vegas, USA, January, 2002.

Guldiken, R, O. \& Degertekin, F, L. (2005). Micromachined Capacitive Transducer Arrays for Intravascular Ultrasound Imaging. Proc. MEMS'05, pp. 315-318, Miami, USA, January, 2005.

Haga, Y.; Fujita, M.; Nakamura, K.; Kim, C, J. \& Esashi, M. (2003). Batch Fabrication of Intravascular Forward-Looking Ultrasonic Probe. Sensors and Actuators A, Vol. 104, pp. 40-43.

Harder, T, A.; Yao, T, J.; He, Q.; Shih, C, Y. \& Tai, Y, C. (2002). Residual Stress in Thin-Film Parylene-C. Proceeding of MEMS'02, pp. 435-438, Las Vegas, USA, January, 2002.

Hayashi, T.; Kawashima, K. \& Endoh, S. (2001). The Generation and Detection of Fundamental Lamb Modes in Plastic Plates by Air-coupled Transducers. Proc. American Institute of Physics Conference, Vol. 557, pp. 105-110, New York, USA, 2001.

Hsieh, W, H.; Yao, T, J. \& Tai, Y, C. (1999). A High Performance MEMS Thin-film Teflon Electret Microphone. Tech. Digest Transducers'99, pp. 1064-1067, Sendai, Japan, June 1999.

Ikeda, M.; Shimizu, N. \& Esashi, M. (1999). Surface Micromachined Driven Shielded Condenser Microphone with a Sacrificial Layer Etched from the Backside. Tech. Digest Transducers'99, pp. 1070-1073, Sendai, Japan, June 1999.

Khuri-Yakub, B, T.; Cheng, C, H.; Degertekin, F, L. \& Ergun, S. (2000). Silicon Micromachined Ultrasonic Transducers. Japanese J. Applied Physics, Vol. 39, pp. 2883-2887.

Knowles Acoustics (2002). Surface Mount Microphones, Knowles Acoustics, Itasca, IL, USA.

Kovacs, G, T, A. (1998). Micromachined Transducers Sourcebook, McGraw-Hill, ISBN 0-07290722-3, New York, USA.

Martin, D, T.; Kadirval, K.; Liu, J.; Fox, R, M.; Sheplak, M. \& Nishida, T. (2005). Surface and Bulk Micromachined Dual Back-Plate Condenser Microphone. Proc. MEMS'05, pp. 319-323, Miami, USA, January 2005. 
Mitsuhashi, W. (1997). Target Parameter Estimation on the basis of Phase Histograms of the Outputs of Constant-Q Filter Bank. IEEJ Trans. Sensors and Micromachines, Vol. 117E, pp. 201-8 (in Japanese).

Mitsuida, Y. (1987). Onkyo Kogaku (Acoustic Engineering), Shokodo, p. 64, ISBN978-4-78560114-0 ,Tokyo, Japan (in Japanese).

Ono, N.; Arita, T.; Senjo,Y. \& Ando, S. (2005). Directivity Steering Principle for Biomimicry Silicon Microphone. Tech. Digest Transducers'05, Vol. 1, pp. 792-795, Seoul, Korea, June 2005.

Pederson, M.; Olthuis, W. \& Bergveld, P. (1998). High-performance Condenser Microphone with Fully Integrated CMOS Amplifier and DC-DC Voltage Converter. J. Microelectromechanical Systems, Vol. 7, No. 4, pp. 387-394.

Sato, H.; Okabe, S. \& Iwata, Y. (1993). Kikai Shindogaku (Mechanical Vibration Theory), Kogyo Chosakai , ISBN 4-7693-2105-8, Tokyo, Japan (in Japanese).

Sasaki, K.; Takano, M. \& Akeno, K. (1988). A New Method of Object Recognition and Sensory Feedback Control by High Accuracy Ultrasonic Sensor. J. The Faculty of Engineering, The University of Tokyo, Ser. B Vol. 49, pp. 209-240.

Scheeper, P, R.; Donk, A, G, H.; Olthuis, W. \& Bergveld, P. (1992). Fabrication of Silicon Condenser Microphones Using Single Wafer Technology. J. Microelectromechanical Systems, Vol. 1, No. 3, pp. 147-154.

Schindel, D, W.; Hutchins, D, A.; Zou, L. \& Sayer, M. (1995). The Design and Characterization of Micromachined Air-Coupled Capacitance Transducers. IEEE Trans. Ultrasonics, Ferroelectrics, and Frequency control, Vol. 42, pp. 42-50.

Škvor, Z. (1967). On the Acoustic Resistance Due to Viscous Losses in Air Gap of Electrostatic Transducers. Acoustica, Vol. 19, pp.295-299.

Tabata, O.; Kawahata, K.; Sugiyama, S. \& Igarashi, I. (1989). Mechanical Property Measurements of Thin Films Using Load-Deflection of Composite Rectangular Membranes. Sensors and Actuators A, Vol. 20, pp. 135-141.

Tai, Y, C. (2003). Parylene MEMS: Material, Technology and Applications. Proc. of 20th Sensor Symposium, pp. 1-8, Tokyo, Japan, October 2003.

Yamashita, K.; Katata, H.; Okuyama, M.; Miyoshi, H.; Kato, G.; Aoyagi, S. \& Suzuki, Y. (2002a). Arrayed Ultrasonic Microsensors with High Directivity for in-Air Use Using PZT Thin Film on Silicon Diaphragms. Sensors and Actuators A, Vol. 97-98, pp. 302-307.

Yamashita, K.; Murakami, H.; Fukunaga, T.; Okuyama, M.; Aoyagi, S. \& Suzuki, Y. (2002b). Ultrasonic Phased Array Micro Sensor Using Piezoelectric PZT Thin Film and Resonant Frequency Tuning by Poling. Proc. 13th IEEE Int. Symp. Applications of Ferroelectrics, pp. 487-490, Nara, Japan, May 2002.

Yao, T, J.; He, Q.; Yang, X. \& Tai, Y, C. (2001). BrF 3 Dry Release Technologies for Large Freestanding Parylene, Tech. Digest. Transducers'01, pp. 652-655, Munich, Germany, June 2001.

Yaralioglu, G, G.; Ergun, A, S. \& Khuri-Yakub, B, T. (2005). Finite-Element Analysis of Capacitive Micromachined Ultrasonic Transducers. IEEE Trans. Ultrasonics, Ferroelectrics, and Frequency control, Vol. 52, pp. 2185-2198. 
Zhuang, X.; Ergun, A, S.; Oralkan, O.; Wygant, I, O. \& Khuri- Yakub, B, T. (2006). Interconnection and Packaging for 2D Capacitive Micromachined Ultrasonic Transducer Arrays Based on Through-Wafer Trench Isolation, Proc. MEMS'06, pp. 270-273, Istanbul, Turk, January, 2006. 


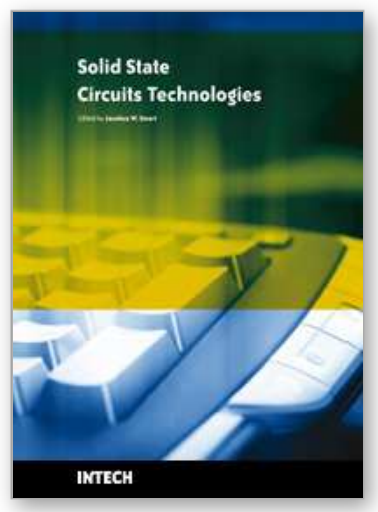

\author{
Solid State Circuits Technologies \\ Edited by Jacobus W. Swart
}

ISBN 978-953-307-045-2

Hard cover, 462 pages

Publisher InTech

Published online 01, January, 2010

Published in print edition January, 2010

The evolution of solid-state circuit technology has a long history within a relatively short period of time. This technology has lead to the modern information society that connects us and tools, a large market, and many types of products and applications. The solid-state circuit technology continuously evolves via breakthroughs and improvements every year. This book is devoted to review and present novel approaches for some of the main issues involved in this exciting and vigorous technology. The book is composed of 22 chapters, written by authors coming from 30 different institutions located in 12 different countries throughout the Americas, Asia and Europe. Thus, reflecting the wide international contribution to the book. The broad range of subjects presented in the book offers a general overview of the main issues in modern solid-state circuit technology. Furthermore, the book offers an in depth analysis on specific subjects for specialists. We believe the book is of great scientific and educational value for many readers. I am profoundly indebted to the support provided by all of those involved in the work. First and foremost I would like to acknowledge and thank the authors who worked hard and generously agreed to share their results and knowledge. Second I would like to express my gratitude to the Intech team that invited me to edit the book and give me their full support and a fruitful experience while working together to combine this book.

\title{
How to reference
}

In order to correctly reference this scholarly work, feel free to copy and paste the following:

Seiji Aoyagi (2010). Micromachined Arrayed Capacitive Ultrasonic Sensor/Transmitter with Parylene Diaphragms, Solid State Circuits Technologies, Jacobus W. Swart (Ed.), ISBN: 978-953-307-045-2, InTech, Available from: http://www.intechopen.com/books/solid-state-circuits-technologies/micromachined-arrayedcapacitive-ultrasonic-sensor-transmitter-with-parylene-diaphragms

\section{INTECH}

open science | open minds

\author{
InTech Europe \\ University Campus STeP Ri \\ Slavka Krautzeka 83/A \\ 51000 Rijeka, Croatia \\ Phone: +385 (51) 770447 \\ Fax: +385 (51) 686166 \\ www.intechopen.com
}

\author{
InTech China \\ Unit 405, Office Block, Hotel Equatorial Shanghai \\ No.65, Yan An Road (West), Shanghai, 200040, China \\ 中国上海市延安西路65号上海国际贵都大饭店办公楼 405 单元 \\ Phone: +86-21-62489820 \\ Fax: +86-21-62489821
}


(C) 2010 The Author(s). Licensee IntechOpen. This chapter is distributed under the terms of the Creative Commons Attribution-NonCommercialShareAlike-3.0 License, which permits use, distribution and reproduction for non-commercial purposes, provided the original is properly cited and derivative works building on this content are distributed under the same license. 